\title{
Patient-reported outcome measures for non-specific neck pain validated in the Italian-language: a systematic review
}

\author{
Leonardo Pellicciari ${ }^{1 *}$, Francesca Bonetti ${ }^{2}$, Damiano Di Foggia ${ }^{3}$, Mauro Monesi ${ }^{4}$ and Stefano Vercelli ${ }^{5}$
}

\begin{abstract}
Background: Patient-reported outcome measures can improve the management of patients with non-specific neck pain. The choice of measure greatly depends on its content and psychometric properties. Most questionnaires were developed for English-speaking people, and need to undergo cross-cultural validation for use in different language contexts. To help Italian clinicians select the most appropriate tool, we systematically reviewed the validated Italian-language outcome measures for non-specific neck pain, and analyzed their psychometric properties and clinical utility.

Methods: The search was performed in MEDLINE, EMBASE, CINAHL, Scopus, Web of Science, and Cochrane Library. All articles published in English or Italian regarding the development, translation, or validation of patient-reported outcome measures available in the Italian language were included. Two reviewers independently selected the studies, extracted data, and assessed methodological quality using the COSMIN checklist.

Results: Out of 4891 articles screened, 66 were eligible. Overall, they were of poor or fair methodological quality. Four instruments measuring function and disability (Neck Disability Index, Neck Pain and Disability Scale, Neck Bournemouth Questionnaire, and Core Outcome Measures Index), and one measuring activity-related fear of movement (NeckPix@) were identified. Each scale showed some psychometric weaknesses or problems with functioning, and none emerged as a gold standard.

Conclusions: Several patient-reported outcome measures are now available for assessing Italian people with non-specific neck pain. While the Neck Disability Index is the one most widely used, the Neck Bournemouth Questionnaire appears the most promising tool from a psychometric point of view.
\end{abstract}

Keywords: Outcome assessment, Quality of life, Spine, Pain, Disability evaluation

\section{Background}

Non-specific neck pain (NSNP) has a multifactorial etiology and it is frequently associated with psychosocial disorders such as anxiety or depression [1]. NSNP affects about two-thirds of people at some stage in their life, especially in middle age [2]. Reliable and valid patientreported outcome measures (PROMs) can provide useful information for a more appropriate prognosis and management. The selection of a PROM greatly depends on its content (the construct being measured), and the

\footnotetext{
* Correspondence: leonardo.pellicciari@gmail.com

'Program in Advanced Sciences and Technologies in Rehabilitation and

Sports Medicine, Tor Vergata University, Rome, Italy

Full list of author information is available at the end of the article
}

soundness of its psychometric properties. These include reliability, validity, responsiveness, interpretability of scores, quality of translation, and acceptable patient/ investigator burden [3].

Several instruments are currently available to assess patients affected by NSNP. A recent review [4] concluded that there was no need for the development of new questionnaires, but rather for more information on the measurement properties of the existing instruments. In most cases, these tools were developed and validated in English-speaking populations. To adapt them to a different language context, a cross-cultural translation process using well-accepted methodological standards is required. In 2011, a systematic review [5] of non-English 
versions of NSNP questionnaires pointed out that the only instrument validated in the Italian language was the Neck Pain and Disability Scale (NPDS). However, in the last 5 years other instruments have been translated or newly developed in Italian, and further studies carried out on the NPDS.

The aim of this study was to systematically review the psychometric properties and clinical utility of the validated Italian-language PROMs available to assess patients affected by NSNP, with the intention of helping clinicians to select the most appropriate scale for their needs.

\section{Methods}

\section{Search strategy and study selection}

A structured search of MEDLINE, CINAHL, EMBASE, Scopus, Web of Science, and Cochrane Library databases was performed from their inception to November 2015. Search strategies for all databases are reported in Appendix. All peer-reviewed articles published in English or Italian that made reference to the development, validation, or clinical use of PROMs to assess patients with NSNP were considered. Other descriptive articles (reviews, clinical trials, letters, commentaries, etc.) that did not provide psychometric data, as well as studies including subjects with specific neck pain (i.e. myelopathy, radiculopathy, whiplash-associated disorders), were excluded.

Three reviewers (FB, DDF, and MM) independently screened titles and abstracts to exclude duplicates and obviously irrelevant studies. The electronic search was complemented by a hand search of the reference list of retrieved articles for additional relevant studies. Disagreements between reviewers were resolved by consensus. Afterwards, two reviewers (LP and SV) independently extracted data on the PROMs available in Italian. For an in-depth understanding of their psychometric properties, data were also collected for any other language version of selected instruments.

\section{Quality assessment}

Methodological quality assessment of the studies included was performed with the COnsensus-based Standards for the selection of health Measurement INstruments (COSMIN) checklist [6]. In the COSMIN checklist ten boxes can be used to assess whether a study meets the standards for good methodological quality. Nine of these boxes contain standards for the included measurement properties and were rated in this review (the Box for criterion validity was excluded as no gold standard exists for neck pain PROMs). Each box consists of different items, that are rated individually on a 4-point rating scale (i.e. "poor", "fair", "good" or "excellent", see http://www.cosmin.nl). Subsequently, an overall score for the assessment of a given measurement property is obtained by taking the lowest score for any of the items in the box ('worst score counts' method). In addition, the generalizability box was used in a data extraction form: information about the characteristics of the study sample in which the measurement properties were assessed are included in the tables related to each scale. Assessment of methodological quality was carried out by two reviewers (LP \& SV) independently. In the case of disagreement, a consensus was obtained through discussion and a third reviewer (FB) gave the score. When the terminology used in the included studies was uncertain, the COSMIN consensus-based definitions of measurement properties were used to decide which properties were assessed and the corresponding boxes to tick.

\section{Data extraction and analysis}

Two authors independently extracted data regarding language, sample size, and studied population. After the assessment of methodological quality with the COSMIN checklist, relevant data on the psychometric properties of reliability, validity, and responsiveness based on classical test theory (CTT) were extracted and interpreted using the following methods [3].

Reliability includes internal consistency and test-retest reliability [7]. The internal consistency is the level of interrelatedness between each item or between items and the total score. A positive rating for internal consistency was given when factor analysis was applied, and Cronbach's alpha was between 0.70 and 0.95 [7]. A low Cronbach's alpha indicates a lack of correlation between the items, which makes summarizing them unjustified, while a very high value indicates redundancy of one or more items [7]. Test-retest reliability concerns the degree to which several measurements made at different times provide similar scores, considering the fact that the clinical condition remains stable. As a general guideline, Intraclass Coefficient Correlation (ICC) values above 0.75 are indicative of good reliability, and those below 0.75 poor to moderate reliability. However, for most clinical measurements reliability should exceed 0.90 in order to ensure reasonable validity [8].

The most common approach used for validation of an instrument is factor analysis [8]. A factor represents a subset of items that are related to each other - but not to items in other factors - reflecting a single theoretical component of the construct (unidimensionality). Unidimensionality of a PROM is a necessary prerequisite to calculate a composite total score. When available, the factor analysis for each PROM was discussed. The construct validity of a scale could be evaluated also in terms of how its score correlates to other measures of the same (convergent validity) and different (divergent validity) constructs [7, 9]. Pearson or Spearman correlations were categorized as strong if $\geq 0.70$, moderate if $0.50-0.69$ and weak if $0.26-0.49$ [10]. 
Responsiveness is the ability of a measure to detect within-person changes over time. Distribution and anchor-based methods are the two general approaches used to interpret score changes and to calculate the Minimal Clinically Important Difference (MCID), also known as the Minimal Important Change [11]. The MCID should be based primarily on anchor-based procedures (Receiver Operating Characteristic [ROC] curves are the preferred approach) [12]; it should be higher than Minimum Detectable Change (MDC) values (the boundary of variability typically found in stable patients) $[12,13]$; and it should not be based on one study or method only [14]. The ROC curve gives the optimal cutoff value (usually the point that jointly maximizes sensitivity and specificity, associated with the least amount of misclassification) and the Area Under the Curve (AUC). The greater the AUC, the greater a measure's ability to distinguish patients who have improved from those who have not improved. As a rule, AUC values between 0.7 and 0.8 are considered as acceptable, and an AUC value higher than 0.8 has a good to excellent discriminative capacity [15]. Among the distribution-based methods, the most useful index is the MDC, i.e. the smallest change in score that is beyond random error. This value represents the statistical significance of individual changes and is expressed in the same metric as the scale. Other indices such as Effect Size (ES), Standardized Response Mean (SRM), or Guyatt's Responsiveness Index (GRI) - are frequently interpreted with Cohen's thresholds: >0.80 large; $>0.50$ moderate; $>0.20$ small [8].

When available, the results of more powerful statistical approaches such as Rasch analysis (RA) were reviewed. Instruments that fit the Rasch model fulfill the requirements for the main mathematic manipulations of the scores, which is a key aspect when measuring clinical changes. RA is being increasingly used in the development and evaluation of PROMs in order to test whether the properties of a questionnaire comply with a wide range of psychometric requirements, such as assessment of response format, item content, appropriate targeting, reliability, and so on [16-18]. RA is used also to provide further confirmation of a scale's unidimensionality. To confirm unidimensionality, a cut-off of $50 \%$ of the variance explained by the Rasch factor (latent trait), and an eigenvalue of the first residual factor $<3$ are usually required conditions [19].

\section{Results}

\section{Study selection}

A total of 4891 articles were initially identified in the literature search. Of these, 118 full-text articles were retrieved and 64 met the inclusion criteria. Two additional articles were found by hand searching. Therefore, a total of 66 articles were included in this systematic review for data collection. A flow chart of the selection process is reported in Fig. 1.

A total of 5 scales or questionnaires were identified: the Neck Disability Index (NDI), the Neck Pain and Disability Scale (NPDS), the Neck Bournemouth Questionnaire (NBQ), the Core Outcome Measures Index (COMI), and NeckPix ${ }^{\odot}$.

\section{Quality assessment}

A detailed methodological assessment of the studies included in the review is reported in Table 1. Overall, most of the psychometric properties were classified as of low (poor to fair) methodological quality. The most common methodological shortcomings found were inadequate sample size included in the analysis, missing information (e.g. percentage of missing items not reported, no description of how missing items were handled), and methodological limitations of specific psychometric properties (i.e. not formulating a priori hypotheses regarding correlations or mean differences, or the direction of correlations or mean differences concerning the hypotheses testing; not complying with all the required translation steps for cross-cultural validity; not formulating a priori hypotheses about the changes in scores and the expected direction of correlations or mean differences of the change scores of PROM regarding responsiveness). Excellent rating was given to only a few boxes, and it was mostly related to the characteristics of internal consistency or validity. A comparison of how instruments validated in Italian language performed with respect to those validated in other languages was not possible owing to the very limited data available on Italian instruments. Cross-cultural validation processes were mainly conducted by a single workgroup. Generally the methodological quality of the translation process was low [20-22], except for the study on NBQ which was good [23]. However, the Italian studies added relevant insights with some good to excellent quality assessment rating, such as for the responsiveness box in the study by Monticone et al. [24].

\section{Data extraction and analysis}

Among the 66 studies included in this review, seven were conducted in Italy. Data regarding language, sample size, and studied population were classified by instrument and are reported in Tables 2, 3, 4, 5 and 6. The most studied psychometric parameters were reliability and validity, while less than half of the studies addressed measurement error and responsiveness. The overall low (poor to fair) quality of the studies and the heterogeneity of statistical approaches used prevented the use of a structured analysis relating results on specific parameters of each instrument to the study's quality. Hence, only a descriptive synthesis of data was possible for each of the five instruments. 


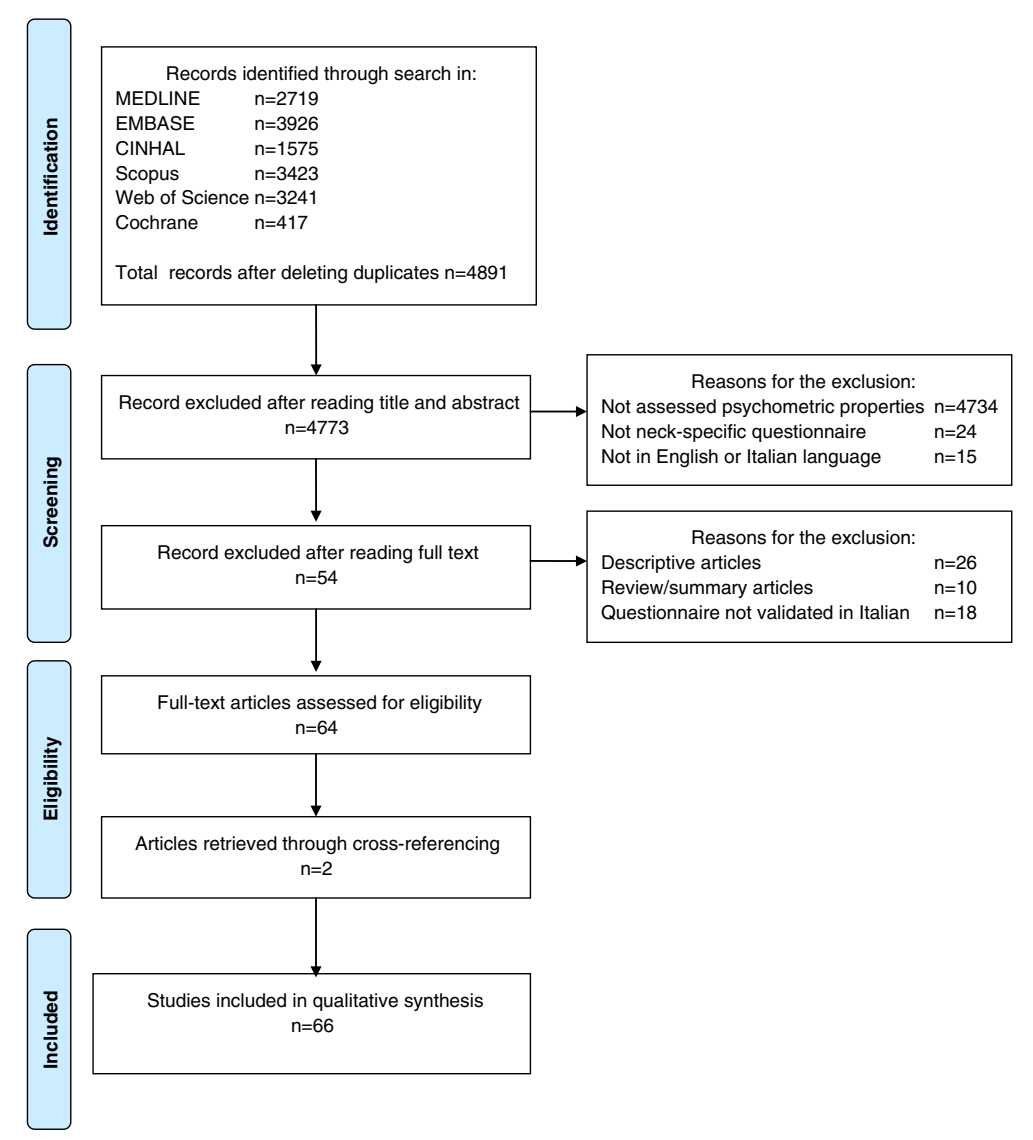

Fig. 1 Flow-chart of study selection

\section{Neck disability index}

The NDI [25] was adapted from an existing questionnaire for low back pain (the Oswestry Disability Index) to assess neck pain and disability. It contains ten items exploring pain intensity, personal care, lifting, reading, headaches, concentration, work, driving, sleeping and recreation. Each item is scored from 0 (no disability) to 5 (worst disability). The total score is calculated by adding the scores of each item and ranges from 0 to 50 , although it is also frequently normalized to 100 or reported as a percentage. The NDI has been translated into many languages [26-52], including Italian [21] (Table 2). The time needed to administer the questionnaire is about 5 to $10 \mathrm{~min}[21,28,36,41,51]$.

Different opinions exist on what the NDI aims to measure and how scores should be interpreted. Although the NDI was mostly considered as a one-factor measure of functional status [33, 34, 37, 40-42, 48, 49, 53-55], other studies [28, 43, 45, 47, 52] -including two of excellent methodological quality $[21,51]$ - suggested the likely presence of sub-dimensions and considered the scale as a measure of pain and disability. According to RA, to achieve unidimensionality some items would need to be removed, but there is no agreement about which (and how many) to remove [44, 46, 56-58]. For example, Johansen et al. [46] proposed a 7-item NDI with a single underlying dimension of disability. They claimed that after removing body function items (\#1 pain, \#5 headache, and \#9 sleep problems), the remaining items - representing the International Classification of Functioning Disability and Health (ICF) component of Activities and Participation - fitted the Rasch model. Suggestions for item reduction ranged from 1 [44] to 5 items [58].

The raw score to measure correlation was poor, indicating that summing of the raw scores is not acceptable and meaningful [56]. The NDI raw score is not linear, and it does not carry with it a clear interpretation of what a score means. Internal consistency was found to be high, ranging from 0.72 [59] to 0.99 [39]. The questionnaire proved to be reliable in most (with ICC values ranging from 0.81 to 0.99 ) [27, 45, 48] but not all studies $[60,61]$, that reported very low reliability values. All of these studies were of poor to fair quality and no firm conclusions can be drawn.

The NDI total score showed moderate to strong correlations with the Visual Analogue Scale for pain (VAS) $[28,31,32,34,38,42,50,53]$, Numeric Rating Scale 
Table 1 Assessment of methodological quality of the included studies using the COnsensus-based standards for the selection of health measurement instruments checklist. Where the psychometric properties were not included in the studies, the boxes are left blanks

\begin{tabular}{|c|c|c|c|c|c|c|c|c|}
\hline Authors, year & $\begin{array}{l}\text { Internal } \\
\text { consistency }\end{array}$ & Reliability & $\begin{array}{l}\text { Measurement } \\
\text { error }\end{array}$ & $\begin{array}{l}\text { Content } \\
\text { validity }\end{array}$ & $\begin{array}{l}\text { Structural } \\
\text { validity }\end{array}$ & $\begin{array}{l}\text { Hypotheses } \\
\text { testing }\end{array}$ & $\begin{array}{l}\text { Translation } \\
\text { process }\end{array}$ & Responsiveness \\
\hline Hains et al, 1998 [53] & Excellent & & & & Good & Good & & \\
\hline Stratford et al, 1999 [54] & Fair & Poor & Poor & & Poor & Poor & & Poor \\
\hline Wheeler et al, 1999 [65] & Poor & & & Poor & Fair & Fair & & \\
\hline Chok \& Gomez, 2000 [26] & & Poor & & & & & & Poor \\
\hline Ackelman \& Lindgren, 2002 [27] & & Poor & & Poor & & Poor & & \\
\hline Bolton \& Humphreys, 2002 [76] & Poor & Poor & Poor & & & Poor & & \\
\hline Goolkasian et al, 2002 [73] & & Poor & & & & Poor & & \\
\hline Wlodyka-Demaille et al, 2002 [28] & & Poor & & & Fair & Fair & Poor & \\
\hline Bicer et al, 2004 [69] & Poor & & & & & Poor & Poor & \\
\hline Bolton, 2004 [80] & & & & & & & & Poor \\
\hline White et al, 2004 [81] & & Fair & & Poor & & Fair & & \\
\hline Wlodyka-Demaille et al, 2004 [75] & & & & & & & & Poor \\
\hline Lee et al, 2006 [29] & Poor & Poor & & & & Poor & Poor & Poor \\
\hline Vos et al, 2006 [30] & & Fair & Fair & & & & & Poor \\
\hline Gay et al, 2007 [59] & Poor & & & & & Poor & & Poor \\
\hline Kose et al, 2007 [31] & Poor & Fair & & & & Poor & Poor & Poor \\
\hline Mousavi et al, 2007 (NDI) [32] & Fair & Fair & & Poor & & & Poor & \\
\hline Mousavi et al, 2007 (NPDS) [32] & Fair & Fair & & Poor & Fair & & Poor & \\
\hline Pool et al, 2007 [63] & & & Fair & & & & & Fair \\
\hline Cleland et al, 2008 [60] & & Fair & Fair & & & & & Fair \\
\hline Kovacs et al, 2008 [82] & Poor & Poor & & & & Poor & Poor & Poor \\
\hline Monticone et al, 2008 [20] & Fair & Fair & & & Fair & Poor & Poor & \\
\hline Scherer et al, 2008 [67] & Excellent & & & & Good & Good & Poor & \\
\hline Trouli et al, 2008 [33] & Good & Poor & Poor & & Good & & Fair & Fair \\
\hline Chan et al, 2009 [74] & & & & Poor & & Poor & & \\
\hline Martel et al, 2009 [77] & & Poor & & & & Fair & Poor & Fair \\
\hline Telci et al, 2009 [36] & & Fair & & & & Poor & Poor & \\
\hline van der Velde et al, 2009 [57] & Fair & & & & Fair & Poor & & \\
\hline Young et al, 2009 [61] & & Poor & Poor & & & & & Good \\
\hline Andrade Ortega et al, 2010 [34] & Fair & Poor & Poor & & Fair & Fair & & Fair \\
\hline Jorritsma et al, 2010 (NDI) [70] & & Poor & & Poor & & & & \\
\hline Jorritsma et al, 2010 (NPDS) [70] & & Poor & Poor & Poor & & & Fair & \\
\hline Salo et al, 2010 [37] & Excellent & Poor & & & Good & Poor & Poor & \\
\hline Wu et al, 2010 (NDI) [38] & Poor & Poor & & & & Poor & Fair & \\
\hline Wu et al, 2010 (NPDS) [38] & Poor & Poor & & & Poor & Poor & Fair & \\
\hline Blozik et al, 2011 [72] & & & Poor & & & & & Poor \\
\hline Chen et al, 2011 [68] & Good & Fair & & & Good & Poor & Fair & \\
\hline Odole et al, 2011 [35] & & Fair & & Poor & & & & \\
\hline Ono et al, 2011 [71] & Fair & Fair & & & Fair & & & Poor \\
\hline Pickering et al, 2011 (NDI) [55] & & & & & Fair & & & \\
\hline Pickering et al, 2011 (NPDS) [55] & & & & & Poor & & & \\
\hline
\end{tabular}

Shakil et al, 2011 [39] 
Table 1 Assessment of methodological quality of the included studies using the COnsensus-based standards for the selection of health measurement instruments checklist. Where the psychometric properties were not included in the studies, the boxes are left blanks (Continued)

\begin{tabular}{|c|c|c|c|c|c|c|c|c|}
\hline Uthaikhup et al, 2011 [40] & Good & Poor & Good & Poor & Good & Poor & Fair & \\
\hline Kesiktas et al, 2012 [41] & Fair & Fair & & & Fair & Fair & Fair & \\
\hline Jorritsma et al, 2012a [62] & Poor & & & Poor & & Good & & \\
\hline Jorritsma et al, 2012b [64] & & & & & & & & Poor \\
\hline Luksanapruksa et al, 2012 [42] & Poor & Fair & & & Poor & Poor & Poor & \\
\hline Monticone et al, 2012 [21] & Excellent & Fair & Good & Poor & Excellent & Good & Fair & \\
\hline Nakamaru et al, 2012 [43] & Poor & Fair & & & Fair & Poor & Fair & Poor \\
\hline Soklic et al, 2012 [78] & Poor & Poor & Poor & & & Poor & Poor & \\
\hline Ailliet at al, 2013 [44] & & & & Excellent & Fair & & Good & \\
\hline Guzy et al, 2013 [45] & Fair & Fair & Fair & & Fair & Poor & Poor & Poor \\
\hline Johansen et al, 2013 [46] & & & & & Good & Poor & & \\
\hline Shaheen et al, 2013 [47] & Poor & Fair & & & Fair & & Fair & Poor \\
\hline Walton \& MacDermid, 2013 [58] & & Fair & Fair & & Fair & Poor & & Poor \\
\hline Cramer et al, 2014 [48] & Fair & Poor & & & Fair & Poor & & Poor \\
\hline Geri et al, 2014 [23] & Good & & & Fair & Good & Good & Good & Good \\
\hline Johansen et al, 2014 [89] & Poor & Fair & Fair & & & & & Poor \\
\hline Miekisiak et al, 2014 [83] & & Good & Good & & Excellent & Poor & & \\
\hline Monticone et al, 2014 [22] & & Good & Good & & & Good & Poor & Poor \\
\hline Monticone et al, 2014 [84] & Excellent & Good & & Poor & Excellent & Good & & \\
\hline Swanenburg et al, 2014 [52] & Poor & Fair & Fair & & Poor & Poor & Fair & \\
\hline Ailliet et al, 2015 [90] & & Fair & Fair & & & & & Poor \\
\hline Bakhtadze et al, 2015 [51] & Excellent & Good & Good & & Excellent & Poor & Fair & \\
\hline Cruz et al, 2015 [49] & Good & Good & & & Good & Poor & Fair & \\
\hline Geri et al, 2015 [79] & Fair & & & & Fair & & & \\
\hline Hung et al, 2015 [56] & & & & & Fair & & & \\
\hline Joseph et al, 2015 [50] & Poor & Fair & & Fair & & Poor & Fair & \\
\hline Monticone et al, 2015 [24] & & & & & & & & Excellent \\
\hline Pereira et al, 2015 [91] & & & Fair & & & & & Fair \\
\hline
\end{tabular}

(NRS) [46, 58], Short Form-36 (SF-36) subscales [27], and other neck disability questionnaires such as NBQ [59] and NPDS [21, 32, 38, 62]. A ceiling and a floorceiling effect was also reported $[30,53,56]$.

Responsiveness was highly affected by the measurement error, as shown also by the very low reliability values reported $[60,61]$. Anchor-based methods gave a MCID ranging from 3.5 [63, 64] (including one study from Italy of excellent quality [24]) to 9.5 [60] points on a 50 -point scale, but the $\mathrm{MDC}_{95}$ showed a very large variability ranging from 1.66 [30] to 23.3 points [60] in studies of fair quality. Accordingly, the amount of change perceived as important by patients is less than $20 \%$ of the maximal total score, but the error of the scale can theoretically reach nearly $50 \%$ of the score.

\section{Neck pain and disability scale}

The NPDS was developed [65] to measure neck pain and disability using the Million Visual Analogue Scale [66] as a template. It consists of 20 items measuring the intensity of pain, its interference with vocational, recreational, social and functional aspects of living, and the presence and extent of associated emotional factors. Each item is rated from 0 to 5 on a $10 \mathrm{~cm}$ VAS divided into 5 equal intervals by vertical bars. Midpoints for each interval are marked with two dots. The total NPDS score is the sum of the scores for all 20 items, ranging from 0 (no disability) to 100 (greatest disability). The maximum acceptable number of missing answers is 4 $[67,68]$. The NPDS has been validated in several languages $[28,29,31,32,38,40,67-71]$, including Italian [20] (Table 3). 
Table 2 Psychometric properties of the neck disability index

\begin{tabular}{|c|c|c|c|c|c|c|c|c|}
\hline \multirow{2}{*}{$\begin{array}{l}\text { Authors, year } \\
\text { (language) }\end{array}$} & \multirow[t]{2}{*}{ Population } & \multirow{2}{*}{$\begin{array}{l}\text { Sample } \\
\text { size }\end{array}$} & \multirow[t]{2}{*}{ Dimensionality } & \multirow{2}{*}{$\begin{array}{l}\text { Internal } \\
\text { consistency }\end{array}$} & \multirow[t]{2}{*}{ Reliability } & \multirow[t]{2}{*}{ Validity } & \multicolumn{2}{|l|}{ Responsiveness } \\
\hline & & & & & & & $\begin{array}{l}\text { Distribution-based } \\
\text { methods }\end{array}$ & $\begin{array}{l}\text { Anchor-based } \\
\text { methods }\end{array}$ \\
\hline $\begin{array}{l}\text { Hains et al, } 1998 \\
\text { (English) [53] }\end{array}$ & $N P$ & 237 & 1 factor & $a=.92$ & - & $r_{p}=.65$ VAS-P & - & - \\
\hline $\begin{array}{l}\text { Stratford et al, } \\
1999 \\
\text { (English) [54] }\end{array}$ & NP & 50 & 1 factor & $a=.87$ & $\begin{array}{l}\text { ICC }=.94 \text { (1-sided } \\
\text { lower } 95 \% \text { Cl .87) }\end{array}$ & $\begin{array}{l}r=.70 \text { prognostic } \\
\text { ratings of change }\end{array}$ & $\begin{array}{l}\mathrm{MDC}=4.7 \text { points } \\
\mathrm{SEM}=2 \text { points }\end{array}$ & $\begin{array}{l}\text { MCID }=5 \text { points } \\
\text { AUC }=.90 \text { (Spec } \\
.80 ; \text { Sens } .78)\end{array}$ \\
\hline $\begin{array}{l}\text { Chok \& Gomez, } 2000 \\
\text { (Malaysian) [26] }\end{array}$ & NP & 22 & - & - & $k=.90$ & - & - & - \\
\hline $\begin{array}{l}\text { Ackelman \& Lindgren, } \\
2002 \text { (Swedish) [27] }\end{array}$ & $\begin{array}{l}\text { Chronic NP } \\
>3 \text { months }\end{array}$ & 39 & - & - & $r_{s}=.94-.99$ & $\begin{array}{l}r_{\mathrm{s}}=.95 \mathrm{DRI} \\
\mathrm{r}_{\mathrm{s}}=-.88 \mathrm{SF}-36 \mathrm{PF} \\
\text { subscale } \\
\mathrm{r}_{\mathrm{s}}=.86 \text { VAS-A } \\
r_{\mathrm{s}}=.60 \text { VAS-P }\end{array}$ & - & - \\
\hline $\begin{array}{l}\text { Wlodyka-Demaille et al, } \\
2002 \text { (French) [28] }\end{array}$ & $\begin{array}{l}\text { Neck disorders } \\
>15 \text { days }\end{array}$ & 101 & $\begin{array}{l}2 \text { factors: Function } \\
\text { and disability; Neck } \\
\text { pain }\end{array}$ & - & $\mathrm{ICC}=.93$ & $\begin{array}{l}r_{s}=.88 \mathrm{NPQ} \\
r_{\mathrm{s}}=.79 \mathrm{NPDS} \\
\mathrm{r}_{\mathrm{s}}=.54 \mathrm{HADS}-\mathrm{D} \\
\mathrm{r}_{\mathrm{s}}=.50 \mathrm{VAS}-\mathrm{D} \\
\mathrm{r}_{\mathrm{s}}=.48 \mathrm{VAS}-\mathrm{P} \\
\mathrm{r}_{\mathrm{s}}=.43 \mathrm{HADS}-\mathrm{A} \\
\mathrm{r}_{\mathrm{s}}=-.41 \text { ROM (Flex-Ext) }\end{array}$ & - & - \\
\hline $\begin{array}{l}\text { Wlodyka-Demaille et al, } \\
2004 \text { (French) [75] }\end{array}$ & $\begin{array}{l}\text { Neck disorders } \\
>15 \text { days }\end{array}$ & 71 & - & - & - & - & $\begin{array}{l}\mathrm{ES}=.55 \\
\mathrm{SRM}=.55\end{array}$ & - \\
\hline $\begin{array}{l}\text { Lee et al, 2006 } \\
\text { (Korean) [29] }\end{array}$ & $N P$ & 180 & - & $a=.92$ & $\begin{array}{l}\mathrm{ICC}_{2,1}=.90(95 \% \\
\mathrm{Cl} .81-.94)\end{array}$ & - & $\begin{array}{l}E S=1.04(84 \% \\
C I .94-1.13) \\
S R M=1.17(84 \% \\
C l 1.06-1.28)\end{array}$ & $\begin{array}{l}\mathrm{AUC}=.79(95 \% \\
\mathrm{Cl} .72-.86)\end{array}$ \\
\hline $\begin{array}{l}\text { Vos et al, } 2006 \\
\text { (Dutch) [30] }\end{array}$ & $\begin{array}{l}\text { First time or } \\
\text { recurrent NP }\end{array}$ & 79 & - & - & $\begin{array}{l}\text { ICC }=.90(95 \% \\
C I .82-.95) \\
\text { LoA }=-7.40-+7.92\end{array}$ & - & $\begin{array}{l}\mathrm{MDC}=1.66 \text { points } \\
\mathrm{SEM}=0.60 \text { points } \\
\text { Guyatt }=1.82\end{array}$ & \\
\hline $\begin{array}{l}\text { Gay et al, } 2007 \\
\text { (English) [59] }\end{array}$ & $N P>3$ months & 23 & - & $a=.72-.77$ & - & $\begin{array}{l}r_{\mathrm{s}}=.77-.80 \mathrm{NBQ} \\
\mathrm{r}_{\mathrm{s}}=.44-.45 \mathrm{VAS}-\mathrm{P}\end{array}$ & $\begin{array}{l}\mathrm{ES}=1.12 \\
\mathrm{SRM}=1.21\end{array}$ & - \\
\hline $\begin{array}{l}\text { Kose et al, } 2007 \\
\text { (Turkish) [31] }\end{array}$ & $\mathrm{NP}>6$ weeks & 102 & - & $a=.90$ & $\mathrm{ICC}=.86$ & $\begin{array}{l}r=.71 \text { VAS-D } \\
r=.65 \text { VAS-P } \\
r=.58 \text { Pain with palpation } \\
r=.50 \text { Pain with motion } \\
r=-.41 \text { ROM (Flex-Ext) } \\
r=-.41 \text { ROM (Rot) }\end{array}$ & $\mathrm{SRM}=.85-.86$ & - \\
\hline $\begin{array}{l}\text { Mousavi et al, } 2007 \\
\text { (Iranian) [32] }\end{array}$ & $N P$ & 185 & - & $a=.88$ & $\mathrm{ICC}=.97$ & $\begin{array}{l}r_{p}=.86 \text { NPDS } \\
r_{p}=.71 \text { VAS-P } \\
r_{p}=-.70--.36 \text { SF-36 } \\
\text { subscales }\end{array}$ & - & - \\
\hline $\begin{array}{l}\text { Pool et al, } 2007 \\
\text { (Dutch) [63] }\end{array}$ & $\begin{array}{l}\text { NP and/or stiffness } \\
>2 \text { weeks }\end{array}$ & 183 & - & - & - & - & $\mathrm{MDC}=10.5$ points & $\begin{array}{l}\text { MCID = 3.5 points } \\
\text { (Spec .70; Sens .90) }\end{array}$ \\
\hline
\end{tabular}


Table 2 Psychometric properties of the neck disability index (Continued)

\begin{tabular}{|c|c|c|c|c|c|c|c|c|}
\hline $\begin{array}{l}\text { Cleland et al, } 2008 \\
\text { (English) [60] }\end{array}$ & NP & 138 & - & - & $\begin{array}{l}\mathrm{ICC}_{2,1}=.50(95 \% \\
\mathrm{Cl} .51-.87) \\
\mathrm{r}_{\mathrm{p}}=.56\end{array}$ & - & $\begin{array}{l}\mathrm{MDC}=19.6 \text { points } \\
\text { SEM }=8.4 \text { points }\end{array}$ & $\begin{array}{l}\text { MCID }=9.5 \text { points } \\
\text { (Spec .72; Sens } .83) \\
\text { AUC }=.83(95 \% \\
C I .75-.90)\end{array}$ \\
\hline $\begin{array}{l}\text { Trouli et al, } 2008 \\
\text { (Greek) [33] }\end{array}$ & NP & 65 & 1 factor & $a=.85$ & $\begin{array}{l}\mathrm{ICC}=.93(95 \% \\
\mathrm{Cl} .84-.97)\end{array}$ & - & $\begin{array}{l}\mathrm{MDC}=1.78 \text { points } \\
\mathrm{SEM}=.64 \text { points }\end{array}$ & - \\
\hline $\begin{array}{l}\text { Chan et al, } 2009 \\
\text { (English) [74] }\end{array}$ & Chronic NP & 20 & - & - & - & $\begin{array}{l}r_{p}=.86 \text { NPDS } \\
r_{p}=.62 \text { PET }\end{array}$ & - & - \\
\hline $\begin{array}{l}\text { Telci et al, } 2009 \\
\text { (Turkish) [36] }\end{array}$ & Chronic NP & 88 & - & - & $\begin{array}{l}\mathrm{ICC}=.98(\mathrm{Cl} 95 \% \\
=.97-.99)\end{array}$ & $\begin{array}{l}r_{p}=.62 \text { VAS-P } \\
r_{p}=.73 \text { NPDS }\end{array}$ & - & - \\
\hline $\begin{array}{l}\text { van der Velde et al, } \\
2009 \text { (English) [57] }\end{array}$ & Mechanical NP & 521 & $\begin{array}{l}\text { RA: unidimensionality } \\
\text { is achieved by removing } \\
\text { items \#3 lifting, and } \\
\text { \#5 headache }\end{array}$ & - & - & NDI-8: $r_{\mathrm{s}}=.42$ Pain & - & - \\
\hline $\begin{array}{l}\text { Young et al, } 2009 \\
\text { (English) [61] }\end{array}$ & Mechanical NP & 91 & - & - & $\begin{array}{l}\mathrm{ICC} C_{2,1}=.64(95 \% \\
\mathrm{Cl} .19-.84)\end{array}$ & - & $\begin{array}{l}\mathrm{MDC}=10.2 \text { points } \\
\mathrm{SEM}=4.3 \text { points }\end{array}$ & $\begin{array}{l}\text { MCID }=7.5 \text { points } \\
\text { AUC }=.79(95 \% \\
C I .68-89)\end{array}$ \\
\hline $\begin{array}{l}\text { Andrade Ortega et al, } \\
2010 \text { (Spanish) [34] }\end{array}$ & NP & 175 & 1 factor & $a=.89$ & $\begin{array}{l}\text { ICC }=.98(95 \% \\
\text { Cl.98-.99) }\end{array}$ & $\begin{array}{l}r_{p}=.89 \mathrm{NPQ} \\
r_{p}=.65 \mathrm{VAS}-\mathrm{P}\end{array}$ & - & - \\
\hline $\begin{array}{l}\text { Jorritsma et al, } 2010 \\
\text { (Dutch) } \\
\text { [70] }\end{array}$ & NP $>3$ months & 32 & - & - & $\begin{array}{l}\mathrm{ICC}=.84(95 \% \\
\mathrm{Cl} .69-.92 \\
\text { LoA }= \pm 6.5\end{array}$ & - & - & - \\
\hline $\begin{array}{l}\text { Salo et al, } 2010 \\
\text { (Finnish) [37] }\end{array}$ & NP & 101 & 1 factor & $a=.85$ & $\begin{array}{l}\text { ICC }=.94(95 \% \\
\text { Cl.90-.96) }\end{array}$ & $\begin{array}{l}r_{p}=.53 \text { VAS-P } \\
r_{p}=.43 \text { DEPS }\end{array}$ & - & - \\
\hline $\begin{array}{l}\text { Wu et al, 2010 } \\
\text { (Chinese) [38] }\end{array}$ & $N P>3$ months & 125 & - & $a=.89$ & $\mathrm{ICC}=.95$ & $\begin{array}{l}r_{p}=.81 \text { NPDS } \\
r_{p}=-.76 \text { to }-.33 \text { SF-36 } \\
\text { subscales } \\
r_{p}=.75 \text { VAS-P }\end{array}$ & - & - \\
\hline $\begin{array}{l}\text { Odole et al, } 2011 \\
\text { (Nigerian) [35] }\end{array}$ & Mechanical NP & 32 & - & - & $r=.97$ & - & - & - \\
\hline $\begin{array}{l}\text { Pickering et al, } 2011 \\
\text { (English) [55] }\end{array}$ & Mechanical NP & 88 & $\begin{array}{l}1 \text { factor: NP and } \\
\text { dysfunction related } \\
\text { to activities }\end{array}$ & - & - & - & - & - \\
\hline $\begin{array}{l}\text { Shakil et al, } 2011 \\
\text { (Hindi) [39] }\end{array}$ & NP & 148 & - & $a=.99$ & $\mathrm{ICC}=.98$ & - & - & - \\
\hline $\begin{array}{l}\text { Uthaikhup et al, } \\
2011 \text { (Thai) [40] }\end{array}$ & NP & 181 & 1 factor & $a=.85$ & $\mathrm{ICC}=.85$ & $r_{s}=.64$ VAS-P & $\begin{array}{l}\mathrm{MDC}=16.1 \text { points } \\
\mathrm{SEM}=5.8 \text { points }\end{array}$ & - \\
\hline $\begin{array}{l}\text { Monticone et al, } \\
2012 \text { (Italian) [21] }\end{array}$ & $\begin{array}{l}\text { Chronic NSNP } \\
>12 \text { weeks }\end{array}$ & 101 & $\begin{array}{l}2 \text { factors: activity of daily } \\
\text { living ( }(F \# 1) \text {, pain and } \\
\text { concentration (F\#2) }\end{array}$ & $\begin{array}{l}a=.84 \\
F \# 1: a=.82 \\
\text { F\#2: } a=.72\end{array}$ & $\begin{array}{l}\text { ICC }=.85(95 \% \\
\text { Cl.78-.89) } \\
\text { F\#1: ICC =.81 }(95 \% \\
C l .73-.87) \\
\text { F\#2: ICC }=.83 \\
(95 \% \mathrm{Cl} .76-.88)\end{array}$ & $\begin{array}{l}r_{\mathrm{s}}=.69 \mathrm{NPDS} \\
\mathrm{r}_{\mathrm{s}}=.55 \mathrm{HADS}-\mathrm{D} \\
\mathrm{r}_{\mathrm{s}}=.44 \mathrm{NRS} \\
\mathrm{r}_{\mathrm{s}}=.42 \mathrm{HADS}-\mathrm{A}\end{array}$ & $\begin{array}{l}M D C=3 \text { points } \\
F \# 1: M D C=1 \\
F \# 2: M D C=1\end{array}$ & - \\
\hline
\end{tabular}


Table 2 Psychometric properties of the neck disability index (Continued)

\begin{tabular}{|c|c|c|c|c|c|c|c|c|}
\hline $\begin{array}{l}\text { Kesiktas et al, } 2012 \\
\text { (Turkish) [41] }\end{array}$ & NP & $30 / 185$ & 1 factor & $a=.88$ & $\mathrm{ICC}=.87-1.0$ & $\begin{array}{l}r_{\mathrm{s}}=.76 \text { VAS-D } \\
\mathrm{r}_{\mathrm{s}}=.62 \mathrm{HADS}-\mathrm{D} \\
\mathrm{r}_{\mathrm{s}}=.60 \text { VAS-P } \\
\mathrm{r}_{\mathrm{s}}=.58 \text { HADS-A }\end{array}$ & - & - \\
\hline $\begin{array}{l}\text { Jorritsma et al, } 2012 a \\
\text { (Dutch) [62] }\end{array}$ & Chronic NP & 125 & - & $a=.83$ & - & $\begin{array}{l}r_{p}=.77 \text { NPDS } \\
r_{p}=-.63-.34 \text { SF- } 36 \\
\text { subscales } \\
r_{p}=.52 \text { VAS-D } \\
r_{p}=.43 \text { VAS-P }\end{array}$ & - & - \\
\hline $\begin{array}{l}\text { Jorritsma et al, 2012b } \\
\text { (Dutch) [64] }\end{array}$ & Chronic NP & 125 & - & - & - & - & $\begin{array}{l}\text { MDC: } 8.4 \text { points } \\
\text { SEM: } 3.0 \text { points }\end{array}$ & $\begin{array}{l}\text { MCID: } 3.5 \text { points } \\
\text { (Spec .66; Sens .74) } \\
\text { AUC }=.75(95 \% \\
\text { CI .64-.87) }\end{array}$ \\
\hline $\begin{array}{l}\text { Nakamaru et al, } 2012 \\
\text { (Japanese) [43] }\end{array}$ & $\begin{array}{l}\text { NP (acute, subacute } \\
\text { and chronic) }\end{array}$ & 110 & $\begin{array}{l}2 \text { factors: activities, } \\
\text { symptoms }\end{array}$ & $a=.88$ & $\begin{array}{l}\text { ICC }=.91(95 \% \\
\text { Cl. } 82-.85)\end{array}$ & $\begin{array}{l}r_{p}=-.51-.25 \text { SF- } 36 \\
\text { subscales }\end{array}$ & $\begin{array}{l}\mathrm{MDC}=6.8 \text { points } \\
\mathrm{SEM}=2.9 \text { points }\end{array}$ & - \\
\hline $\begin{array}{l}\text { Luksanapruksa et al, } \\
2012 \text { (Thai) [42] }\end{array}$ & $\begin{array}{l}\text { Outpatients } \\
\text { with NP }\end{array}$ & 46 & 1 factor (activities) & $a=.92$ & $\mathrm{ICC}=.99$ & $\begin{array}{l}r_{p}=.89 \text { VAS }-P \\
r_{p}=-.43 \text { SF- } 36\end{array}$ & - & - \\
\hline $\begin{array}{l}\text { Ailliet et al, } 2013 \\
\text { (Dutch) [44] }\end{array}$ & $N P$ & 338 & $\begin{array}{l}\text { RA: unidimensionality } \\
\text { is achieved by removing } \\
\text { item } \# 6 \text { concentration }\end{array}$ & - & - & $r_{p}=.75 \mathrm{DASH}$ & - & - \\
\hline $\begin{array}{l}\text { Guzy et al, } 2013 \\
\text { (Polish) [45] }\end{array}$ & NSNP $>7$ weeks & 95 & 2 factors & $\begin{array}{l}a=.82 \\
\text { F\#1: } a=.77 \\
\text { F\#2: } a=.73\end{array}$ & $\begin{array}{l}\text { ICC }=.99(95 \% \\
C \mathrm{Cl} .98-.99) \\
\text { LoA }= \pm 2.48\end{array}$ & $r_{p}=.55$ VAS $-\mathrm{P}$ & $\begin{array}{l}\mathrm{MDC}=5.96 \text { points } \\
\mathrm{SEM}=2.15 \text { points } \\
\text { SRM }=1.61\end{array}$ & $\begin{array}{l}\text { MCID }=6.5 \text { points } \\
\text { (Spec } .81 ; \text { Sens. } .90) \\
\text { AUC }=.91(95 \% \\
C I .84-.97)\end{array}$ \\
\hline $\begin{array}{l}\text { Johansen et al, } 2013 \\
\text { (Norwegian) [46] }\end{array}$ & $N P$ & 249 & $\begin{array}{l}\text { RA: unidimensionality is } \\
\text { achieved by removing } \\
\text { items \#1 pain, \#5 } \\
\text { headache, and \#9 sleep }\end{array}$ & - & - & $\begin{array}{l}r_{p}=.59 \mathrm{HSCL} \\
r_{p}=.58 \mathrm{NRS} \\
\text { NDI-7: } r_{p}=.56 \mathrm{NRS} \\
r_{p}=.43 \text { TSK } \\
\text { NDI-7: } r_{p}=.54 \mathrm{HSCL}\end{array}$ & - & - \\
\hline $\begin{array}{l}\text { Shaheen et al, } 2013 \\
\text { (Arabic) [47] }\end{array}$ & Neck complaints & 65 & $\begin{array}{l}2 \text { factors: activity of daily } \\
\text { living (F\#1); pain and } \\
\text { concentration (F\#2) }\end{array}$ & $\begin{array}{l}a=.89 \\
\text { F\#1: } a=.86 \\
\text { F\#2: } a=.77\end{array}$ & 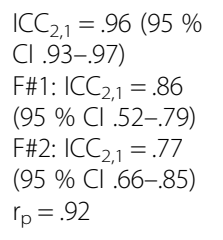 & - & - & - \\
\hline $\begin{array}{l}\text { Walton \& MacDermid, } \\
2013 \text { (English) [58] }\end{array}$ & Mechanical NP & 316 & $\begin{array}{l}\text { RA: unidimensionality is } \\
\text { achieved by removing } \\
\text { items \#1 pain, \#3 lifting, } \\
\text { \#4 reading, \#5 headache, } \\
\text { and \#9 sleep }\end{array}$ & - & $\begin{array}{l}\mathrm{ICC}_{2,1}=.92(95 \% \\
\mathrm{Cl} 85-.96) \\
\mathrm{NDI}-5: \mathrm{ICC} 2,1=.94 \\
(95 \% \mathrm{Cl} .83-.86)\end{array}$ & $\begin{array}{l}r_{p}=.71 \mathrm{NRS} \\
r_{p}=.64 \text { PCS } \\
r_{p}=.53 \text { TSK } \\
\text { NDI-5: } r_{p}=.71 \text { PCS } \\
\text { NDI-5: } r_{p}=.67 \text { NPRS } \\
\text { NDI-5: } r_{p}=.54 \text { TSK }\end{array}$ & $\begin{array}{l}\mathrm{MDC}_{90}=4.5 \text { points } \\
\mathrm{SEM}=1.95 \text { points } \\
\mathrm{ES}=.71 \\
\mathrm{NDI}-5: \mathrm{MDC}_{90}=2.7 \\
\text { points } \\
\mathrm{SEM}=1.15 \text { points } \\
\mathrm{ES}=.85\end{array}$ & $\begin{array}{l}\mathrm{AUC}=.76(95 \% \\
\mathrm{Cl} .63-.89) \\
\mathrm{NDI}-5: \mathrm{AUC}=.72 \\
(95 \% \mathrm{Cl} .59-.86)\end{array}$ \\
\hline $\begin{array}{l}\text { Cramer et al, } 2014 \\
\text { (German) [48] }\end{array}$ & $\begin{array}{l}\text { Chronic NSNP } \\
>12 \text { weeks }\end{array}$ & 558 & 1 factor & $a=.81$ & $\begin{array}{l}\mathrm{ICC}=.81(95 \% \\
\mathrm{Cl} .78-.83)\end{array}$ & $\begin{array}{l}r_{s}=-.45--0.30 \text { SF- } 36 \\
\text { subscales }\end{array}$ & - & - \\
\hline
\end{tabular}


Table 2 Psychometric properties of the neck disability index (Continued)

\begin{tabular}{|c|c|c|c|c|c|c|c|c|}
\hline $\begin{array}{l}\text { Johansen et al, } 2014 \\
\text { (Norwegian) [89] }\end{array}$ & $N P$ & 255 & - & $a=.83-.91$ & $\begin{array}{l}\mathrm{ICC}=.84(95 \% \\
\mathrm{Cl} .72-.91)\end{array}$ & - & $\begin{array}{l}\mathrm{MDC}=6.15 \text { points } \\
\mathrm{SEM}=4.44 \text { points }\end{array}$ & $\begin{array}{l}\text { MCID }=8.3 \text { points } \\
\text { AUC }=.70(95 \% \\
C I .58-.82)\end{array}$ \\
\hline $\begin{array}{l}\text { Swanenburg et al, } \\
2014 \text { (German) [52] }\end{array}$ & $\begin{array}{l}\text { Outpatients } \\
\text { with NP }\end{array}$ & 49 & 2 factors & $a=.96$ & $\begin{array}{l}\mathrm{ICC}_{(2,1)}=.92(95 \% \\
\mathrm{Cl} .84-.96)\end{array}$ & $\mathrm{r}_{\mathrm{s}}=.55$ VAS-P & $\begin{array}{l}\mathrm{MDC}=6.16 \text { points } \\
\mathrm{SEM}=2.22 \text { points }\end{array}$ & - \\
\hline $\begin{array}{l}\text { Hung et al, } 2015 \\
\text { (English) [56] }\end{array}$ & Neck complaints & 865 & $\begin{array}{l}\text { RA: unidimensionality is } \\
\text { achieved by removing } \\
\text { items \#2 personal care, } \\
\text { and } \# 5 \text { headache }\end{array}$ & - & - & - & - & - \\
\hline $\begin{array}{l}\text { Ailliet et al, } 2015 \\
\text { (Dutch) [90] }\end{array}$ & NP & 337 & - & - & $\begin{array}{l}\mathrm{ICC}=.88 \\
\mathrm{LOA}=-5.02-+5.60\end{array}$ & - & $\begin{array}{l}\mathrm{MDC}=5.40 \text { points } \\
\mathrm{SEM}=1.95 \text { points }\end{array}$ & $\begin{array}{l}\mathrm{MCID}=4.50 \text { points } \\
\mathrm{AUC}=.85\end{array}$ \\
\hline $\begin{array}{l}\text { Cruz, } 2015 \text { (Portuguese) } \\
\text { [49] }\end{array}$ & $\begin{array}{l}\text { Chronic NP } \\
\text { (for at least } 3 \\
\text { months) }\end{array}$ & 113 & 1 factor & $a=.95$ & $\begin{array}{l}\mathrm{ICC}=.91(95 \% \\
\mathrm{Cl} .87-.94) \\
\mathrm{LoA}=1.59 \pm 3.70\end{array}$ & $r_{s}=.49 \mathrm{NRS}$ & - & - \\
\hline $\begin{array}{l}\text { Joseph et al, } 2015 \\
\text { (Marathi) [50] }\end{array}$ & NP $>3$ months & 81 & - & $a=.97$ & ICC $=.95$ & $r=.95$ VAS-P & - & - \\
\hline $\begin{array}{l}\text { Monticone et al, } 2015 \\
\text { (Italian) [24] }\end{array}$ & $\begin{array}{l}\text { Outpatients with } \\
\text { chronic NP }\end{array}$ & 200 & - & - & - & - & $\begin{array}{l}\mathrm{ES}=.66 \\
\mathrm{SRM}=1.09 \\
\mathrm{GRI}=.70\end{array}$ & $\begin{array}{l}\mathrm{MCID}=3.5 \text { points } \\
\mathrm{AUC}=.96 \text { (spec } \\
.81 ; \text { sens } .98)\end{array}$ \\
\hline $\begin{array}{l}\text { Bakhtadze et al, } 2015 \\
\text { (Russian) [51] }\end{array}$ & $\begin{array}{l}\text { Acute }(\leq 12 \\
\text { weeks) or chronic } \\
(\geq 12 \text { week) NP }\end{array}$ & $80 / 109$ & 2 factors & $\begin{array}{l}a=.83 \\
F \# 1: a=.82 \\
\text { F\#2: } a=.66\end{array}$ & $\begin{array}{l}\text { ICC }=.91(95 \% \\
\text { Cl.86-.94) }\end{array}$ & $r_{s}=.62$ GRS $-P$ & $\mathrm{MDC}=5.4$ points & \\
\hline $\begin{array}{l}\text { Pereira et al, } 2015 \\
\text { (Portuguese) [91] }\end{array}$ & Chronic NP & 108 & - & - & - & - & $\begin{array}{l}M^{M D C_{95}}=12 \text { points } \\
\text { SEM }=4.27 \text { points }\end{array}$ & $\begin{array}{l}\mathrm{MCID}=5.5 \text { points } \\
\mathrm{AUC}=.59(\text { spec } \\
.57 ; \text { sens } .61)\end{array}$ \\
\hline
\end{tabular}


Table 3 Psychometric properties of the neck pain and disability scale

\begin{tabular}{|c|c|c|c|c|c|c|c|c|}
\hline \multirow{2}{*}{$\begin{array}{l}\text { Authors, year } \\
\text { (language) }\end{array}$} & \multirow[t]{2}{*}{ Population } & \multirow{2}{*}{$\begin{array}{l}\text { Sample } \\
\text { size }\end{array}$} & \multirow[t]{2}{*}{ Dimensionality } & \multirow[t]{2}{*}{ Internal consistency } & \multirow[t]{2}{*}{ Reliability } & \multirow[t]{2}{*}{ Validity } & \multicolumn{2}{|l|}{ Responsiveness } \\
\hline & & & & & & & $\begin{array}{l}\text { Distribution-based } \\
\text { methods }\end{array}$ & $\begin{array}{l}\text { Anchor-based } \\
\text { methods }\end{array}$ \\
\hline $\begin{array}{l}\text { Wheeler et al, } 1999 \\
\text { (English) [65] }\end{array}$ & NP & 100 & $\begin{array}{l}4 \text { factors: neck problems; pain intensity; } \\
\text { effect of neck pain on motion and } \\
\text { cognition; neck pain interference with } \\
\text { life activities }\end{array}$ & $a=.93$ & - & $\begin{array}{l}r_{p}=.78 \mathrm{ODI} \\
r_{p}=.80 \mathrm{PDI} \\
r_{p}=.52 \mathrm{BDI}\end{array}$ & - & - \\
\hline $\begin{array}{l}\text { Goolkasian et al, } 2002 \\
\text { (English) [73] }\end{array}$ & NP & 83 & - & - & $r_{p}=.93$ & $\begin{array}{l}r=.59 \text { Patient GAS } \\
r=.59 \text { Physician GAS } \\
r=.74 \mathrm{PDI} \\
r=.72 \mathrm{NDI}\end{array}$ & - & - \\
\hline $\begin{array}{l}\text { Wlodyka-Demaille et } \\
\text { al, } 2002 \text { (French) [28] }\end{array}$ & NP $>15$ days & 101 & $\begin{array}{l}3 \text { factors: function and disability; neck } \\
\text { pain intensity during movement; static } \\
\text { neck pain intensity }\end{array}$ & - & $\mathrm{ICC}=.91$ & $\begin{array}{l}r_{\mathrm{s}}=.79 \mathrm{NDI} \\
r_{\mathrm{s}}=.73 \mathrm{NPQ} \\
\mathrm{r}_{\mathrm{s}}=.63 \mathrm{VAS}-\mathrm{D} \\
r_{\mathrm{s}}=.51 \mathrm{VAS}-\mathrm{P} \\
r_{\mathrm{s}}=.40 \mathrm{HADS}-\mathrm{A} \\
\mathrm{r}_{\mathrm{s}}=.49 \mathrm{HADS}-\mathrm{D} \\
r_{\mathrm{s}}=-.45 \mathrm{ROM} \\
\text { (Flex-Ext) }\end{array}$ & - & - \\
\hline $\begin{array}{l}\text { Bicer et al, } 2004 \\
\text { (Turkish) [69] }\end{array}$ & NP $>6$ months & 61 & - & $\begin{array}{l}a=.86 \\
\text { Item to total } \\
\text { correlation from } .08 \\
\text { to } .69\end{array}$ & - & $\begin{array}{l}r=.51 \text { PDI } \\
r=.45 \text { VAS-P }\end{array}$ & - & - \\
\hline $\begin{array}{l}\text { Wlodyka-Demaille et } \\
\text { al, } 2004 \text { (French) [75] }\end{array}$ & NP $>15$ days & 71 & - & - & - & - & $\begin{array}{l}\mathrm{ES}=.46 \\
\mathrm{SRM}=.38\end{array}$ & - \\
\hline $\begin{array}{l}\text { Lee et al, } 2006 \\
\text { (Korean) [29] }\end{array}$ & NP & 180 & - & $a=.96$ & $\begin{array}{l}\mathrm{ICC}=.90(95 \% \\
\mathrm{Cl} .83-.95)\end{array}$ & - & $\begin{array}{l}E S=1.07 \\
S R M=1.34\end{array}$ & $\begin{array}{l}\mathrm{A} \cup \mathrm{C}=.79(95 \% \\
\mathrm{Cl} 72-.86)\end{array}$ \\
\hline $\begin{array}{l}\text { Kose et al, } 2007 \\
\text { (Turkish) [31] }\end{array}$ & NP $>6$ weeks & 102 & - & $a=.94$ & $\mathrm{ICC}=.81$ & $\begin{array}{l}r=.40 \text { Morning } \\
\text { stiffness } \\
r=.73 \text { VAS-P } \\
r=.69 \text { VAS-D } \\
r=.46 \text { VAS-Physician's } \\
\text { assessment } \\
r=.49 \text { Pain with } \\
\text { motion }\end{array}$ & $\mathrm{SRM}=.89-.92$ & - \\
\hline $\begin{array}{l}\text { Mousavi et al, } 2007 \\
\text { (Iranian) [32] }\end{array}$ & NP & 185 & $\begin{array}{l}4 \text { factors: neck dysfunction and disability } \\
(\text { F\#1), neck pain intensity (F\#2), neck pain } \\
\text { during movement (F\#3), static neck pain } \\
\text { problems (F\#4) }\end{array}$ & $\begin{array}{l}\text { F\#1: } a=.94 \\
\text { F\#2: } a=.92 \\
\text { F\#3: } a=.84 \\
\text { F\#4: } a=.75\end{array}$ & $\begin{array}{l}\text { F\#1: ICC }=.95 \\
\text { F\#2: ICC }=.97 \\
\text { F\#3: ICC }=.92 \\
\text { F\#4: ICC }=.90\end{array}$ & $\begin{array}{l}\text { F\#1: } r_{p}=-.69 \text { to }-.40 \\
\text { SF-36 subscales } \\
\text { F\#2: } r_{p}=-.57 \text { to }-.24 \\
\text { SF-36 subscales } \\
\text { F\#3: } r_{p}=-.54 \text { to }-.17 \\
\text { SF-36 subscales } \\
\text { F\#4: } r_{p}=-.63 \text { to }-.18 \\
\text { SF-36 subscales } \\
\text { F\#1: } r_{p}=.63 \text { VAS-P } \\
\text { F\#2: } r_{p}=.77 \text { VAS-P } \\
\text { F\#3: } r_{p}=.79 \text { VAS-P } \\
\text { F\#4: } r_{p}=.46 \text { VAS-P }\end{array}$ & - & - \\
\hline
\end{tabular}


Table 3 Psychometric properties of the neck pain and disability scale (Continued)

\begin{tabular}{|c|c|c|c|c|c|c|c|c|}
\hline & & & & & & $\begin{array}{l}\text { Total NPDS: } r_{p}=.86 \\
\text { NDI }\end{array}$ & & \\
\hline $\begin{array}{l}\text { Monticone et al, } 2008 \\
\text { (Italian) [20] }\end{array}$ & $\begin{array}{l}\text { NP subacute (pain } \\
>4 \text { weeks) and } \\
\text { chronic (pain } \\
>12 \text { weeks) }\end{array}$ & 157 & $\begin{array}{l}3 \text { factors: neck dysfunction related to } \\
\text { general activities (F\#1), neck pain and } \\
\text { cognitive-behavioral aspects (F\#2), neck } \\
\text { dysfunction related to activities of the } \\
\text { cervical spine (F\#3) }\end{array}$ & $\begin{array}{l}\text { Total NPDS: } a=.94 \\
\text { F\#1: } a=.92 \\
\text { F\#2: } a=.86 \\
\text { F\#3: } a=.89\end{array}$ & $\begin{array}{l}\text { Total NPDS: } \\
r_{s}=.91 \\
\text { F\#1: } r_{s}=.89 \\
\text { F\#2: } r_{s}=.93 \\
\text { F\#3: } r_{s}=.92\end{array}$ & $\begin{array}{l}r_{p}=-.47 \text { SF- } 36 \\
r_{p}=-.45 \text { to }-.17 \text { SF-36 } \\
\text { subscales }\end{array}$ & - & - \\
\hline $\begin{array}{l}\text { Scherer et al, } 2008 \\
\text { (German) [67] }\end{array}$ & NP & 448 & 3 factors & $\begin{array}{l}a=.94 \\
\text { Item-to-total } \\
\text { correlation from } .46 \\
\text { to } .82\end{array}$ & - & $\begin{array}{l}r_{p}=.44 \text { HADS-D } \\
r_{p}=.41 \text { HADS- } A\end{array}$ & - & - \\
\hline $\begin{array}{l}\text { Chan et al, } 2009 \\
\text { (English) [74] }\end{array}$ & NP & 20 & - & - & - & $\begin{array}{l}r_{p}=.71 \mathrm{PET} \\
r_{p}=.86 \mathrm{NDI}\end{array}$ & - & - \\
\hline $\begin{array}{l}\text { Jorritsma et al, } 2010 \\
\text { (Dutch) [70] }\end{array}$ & NP $>3$ months & 33 & - & - & $\begin{array}{l}\text { ICC }=.76(95 \\
\% \text { Cl } .57-.87) \\
\text { LoA: } \pm 20.9\end{array}$ & - & - & - \\
\hline $\begin{array}{l}\text { Wu et al, 2010 } \\
\text { (Chinese) [38] }\end{array}$ & NP $>3$ months & 125 & $\begin{array}{l}3 \text { factors: neck disfunction and disability } \\
(\text { F\#1), neck pain intensity during movement } \\
(\text { F\#2), static neck pain intensity (F\#3) }\end{array}$ & $\begin{array}{l}\text { F\#1: } \mathrm{a}=.91 \\
\text { F\#2: } \mathrm{a}=.88 \\
\text { F\#3: } \mathrm{a}=.82\end{array}$ & $\begin{array}{l}\text { F\#1: ICC }=.94 \\
\text { F\#2: ICC }=.92 \\
\text { F\#3: ICC }=.86\end{array}$ & $\begin{array}{l}\text { Total NPDS: } r_{p}=.81 \\
\text { NDI } \\
\text { F\#1: } r_{p}=-.61 \text { to }-.20 \\
\text { SF-36 subscales } \\
\text { F\#2: } r_{p}=-.58 \text { to }-.19 \\
\text { SF-36 subscales } \\
\text { F\#3: } r_{p}=-.61 \text { to }-.22 \\
\text { SF-36 subscales } \\
\text { F\#1: } r_{p}=.72 \text { VAS-P } \\
\text { F\#2: } r_{p}=.62 \text { VAS-P } \\
\text { F\#3: } r_{p}=.76 \text { VAS-P }\end{array}$ & - & - \\
\hline $\begin{array}{l}\text { Blozik et al, } 2011 \\
\text { (German) [72] }\end{array}$ & $N P$ & 411 & - & - & - & - & $\begin{array}{l}\mathrm{MDC}=3 \text { points } \\
\mathrm{SEM}=.9 \text { points } \\
\mathrm{SRM}=1.1\end{array}$ & - \\
\hline $\begin{array}{l}\text { Chen et al, } 2011 \\
\text { (Chinese) [68] }\end{array}$ & NP >3 months & 106 & $\begin{array}{l}4 \text { factors: pain (F\#1), disability (F\#2), } \\
\text { Neck specific function (F\#3), emotional } \\
\text { and cognitive influences (F\#4) }\end{array}$ & $\begin{array}{l}\text { Total NPDS: } a=.97 \\
\text { F\#1: } a=.93 \\
\text { F\#2: } a=.95 \\
\text { F\#3: } a=.95 \\
\text { F\#4: } a=.91 \\
\text { Item-to-total } \\
\text { correlation: from } .68 \\
\text { to } .88\end{array}$ & $\begin{array}{l}\text { Total NPDS: } r \\
=.81 \\
\text { F\#1: } r=.89 \\
\text { F\#2: } r=.97 \\
\text { F\#3: } r=.91 \\
\text { F\#4: } r=.94\end{array}$ & $\begin{array}{l}\text { Total NPDS: } r_{p}=-.72 \\
\text { SF-36 } \\
\text { Total NPDS: } r_{p}=-.71 \text { to } \\
-.12 \text { SF-36 subscales } \\
\text { F\#1: } r_{p}=-.74 \text { SF-36 } \\
\text { F\#1: } r_{p}=-.71 \text { to }-.05 \\
\text { SF-36 subscales } \\
\text { F\#2: } r_{p}=-.67 \text { SF-36 } \\
\text { F\#2: } r_{p}=-.65 \text { to }-.14 \\
\text { SF-36 subscales } \\
\text { F\#3: } r_{p}=-.49 \text { SF-36 } \\
\text { F\#3: } r_{p}=-.65 \text { to }-.04 \\
\text { SF-36 subscales } \\
\text { F\#4: } r_{p}=-.63 \text { SF-36 } \\
\text { F\#4: } r_{p}=-.63 \text { to }-.12 \\
\text { SF-36 subscales }\end{array}$ & - & - \\
\hline
\end{tabular}


Table 3 Psychometric properties of the neck pain and disability scale (Continued)

\begin{tabular}{|c|c|c|c|c|c|c|c|c|}
\hline $\begin{array}{l}\text { Ono et al, } 2011 \\
\text { (Japanese) [71] }\end{array}$ & $\mathrm{NP}$ & 167 & $\begin{array}{l}2 \text { factors: neck-pain-related disability } \\
(\mathrm{F \# 1)} \text { and neck-related pain (F\#2) }\end{array}$ & $\begin{array}{l}\text { Total NPDS } a=.96 \\
\text { F\#1: } a=.94 \\
\text { F\#2: } a=.93\end{array}$ & $\begin{array}{l}\text { Total NDPS: } \\
\text { ICC }=.77 \\
\text { F\#1: ICC }=.75 \\
\text { F\#2: ICC }=.77\end{array}$ & $\begin{array}{l}\text { Total NPDS: } r_{p}=-.54 \text { to } \\
-.24 \text { SF-36 subscales } \\
\text { F\#1: } r_{p}=-.50 \text { to }-.24 \\
\text { SF-36 subscales } \\
\text { F\#2: } r_{p}=-.51 \text { to }-.22 \\
\text { SF-36 subscales } \\
\text { Total NPDS: } r_{p}=.71 \\
\text { VAS-P } \\
\text { F\#1: } r_{p}=.56 \text { VAS-P } \\
\text { F\#2: } r_{p}=.77 \text { VAS-P } \\
\text { Total NPDS: } r_{p}=.59 \\
\text { VAS-D } \\
\text { F\#1: } r_{p}=.48 \text { VAS-D } \\
\text { F\#2: } r_{p}=.63 \text { VAS-D }\end{array}$ & - & - \\
\hline $\begin{array}{l}\text { Pickering et al," } \\
2011 \text { (English) [55] }\end{array}$ & $N P$ & 88 & $\begin{array}{l}3 \text { factors: dysfunction related to general } \\
\text { activities and the impact of participation } \\
\text { restriction on psychosocial function; } \\
\text { neck pain and interference with } \\
\text { neck-specific function; cognitive and } \\
\text { emotional functioning }\end{array}$ & - & - & - & - & - \\
\hline $\begin{array}{l}\text { Uthaikhup et al, } 2011 \\
\text { (Thai) [40] }\end{array}$ & NP & 172 & $\begin{array}{l}3 \text { factors: disability (F\#1), pain (F\#2) } \\
\text { neck specific function (F\#3) }\end{array}$ & $\begin{array}{l}\text { Total NDPS: } a=.96 \\
\text { F\#1: } a=.94 \\
\text { F\#2: } a=.90 \\
\text { F\#3: } a=.92\end{array}$ & $\begin{array}{l}\text { Total NDPS: } \\
\text { ICC }=.88 \\
\text { F\#1: ICC }=.81 \\
\text { F\#2: ICC }=.91 \\
\text { F\#3: ICC }=.74\end{array}$ & $\begin{array}{l}\text { Total NPDS: } r_{s}=.76 \\
\text { VAS-P } \\
\text { F\#1: } r_{s}=.74 \text { VAS-P } \\
\text { F\#2: } r_{s}=.65 \text { VAS-P } \\
\text { F\#3: } r_{s}=.61 \text { VAS-P }\end{array}$ & $\begin{array}{l}M D C=21.8 \\
\text { points SEM }=7.9\end{array}$ & - \\
\hline $\begin{array}{l}\text { Jorritsma et al, 2012a } \\
\text { (Dutch) [62] }\end{array}$ & $N P$ & 125 & - & $\begin{array}{l}a=.93 \\
\text { Item-total } \\
\text { correlations from } .45 \\
\text { to } .73\end{array}$ & - & $\begin{array}{l}r_{p}=-.70 \text { to }-.36 \text { SF- } 36 \\
\text { subscales } \\
r_{p}=.54 \text { VAS-P } \\
r_{p}=.57 \text { VAS-D } \\
r_{p}=.77 \text { NDI }\end{array}$ & - & - \\
\hline $\begin{array}{l}\text { Jorritsma et al, 2012b } \\
\text { (Dutch) [64] }\end{array}$ & $N P$ & 125 & - & - & - & - & $\begin{array}{l}\mathrm{MDC}=31.7 \\
\text { points } \mathrm{SEM}=11.4\end{array}$ & $\begin{array}{l}\text { MCID }=11.5 \text { points } \\
\text { (Sens .74; Spec .70) } \\
\text { AUC }=.75(95 \% \\
\text { Cl .62-.87) }\end{array}$ \\
\hline $\begin{array}{l}\text { Monticone et al, } 2015 \\
\text { (Italian) [24] }\end{array}$ & NP & 200 & - & - & - & - & $\begin{array}{l}\mathrm{ES}=.73 \\
\mathrm{SRM}=1.26 \\
\mathrm{GRI}=.73\end{array}$ & $\begin{array}{l}\text { MCID }=10 \text { points } \\
\text { AUC =.91 (Sens } \\
.93 ; \text { Spec .83) }\end{array}$ \\
\hline
\end{tabular}

a Cronbach's alpha, AUC area under the curve, BDI Beck depression inventory, CI confidence interval, Flex flexion, ES effect size, Ext extension, GAS global assessment score, GRI Guyatt's responsiveness index, HADS-A hospital anxiety and depression scale of anxiety, HADS-D hospital anxiety and depression scale of depression, ICC intraclass correlation coefficient, LoA limits of agreement, MCID minimal clinically important difference, MDC minimal detectable change, NDI neck disability index, NP neck pain, NPDS neck pain and disability scale, NPQ northwick park questionnaire, ODI oswestry disability index, $P D I$ pain disability index, PET problem elicitation technique, $r$ correlation coefficient, $r_{p}$ Pearson's correlation coefficient, $r_{s}$ Spearman's correlation coefficient, ROM range of motion, SEM standard error of measurement, Sens sensibility, SF-36 the medical

outcomes study 36 -item short-form health survey, Spec specificity, SRM standardized response mean, VAS-D visual analogue scale for disability, VAS-H visual analogue scale for global health, VAS-P visual analogue scale for pain 
Table 4 Psychometric properties of the neck bournemouth questionnaire

\begin{tabular}{|c|c|c|c|c|c|c|c|c|}
\hline \multirow{2}{*}{$\begin{array}{l}\text { Authors, year } \\
\text { (language) }\end{array}$} & \multirow[t]{2}{*}{ Population } & \multirow{2}{*}{$\begin{array}{l}\text { Sample } \\
\text { size }\end{array}$} & \multirow[t]{2}{*}{ Dimensionality } & \multirow[t]{2}{*}{ Internal consistency } & \multirow[t]{2}{*}{ Reliability } & \multirow[t]{2}{*}{ Validity } & \multicolumn{2}{|l|}{ Responsiveness } \\
\hline & & & & & & & $\begin{array}{l}\text { Distribution-based } \\
\text { methods }\end{array}$ & $\begin{array}{l}\text { Anchor-based } \\
\text { methods }\end{array}$ \\
\hline $\begin{array}{l}\text { Bolton \& Humphreys, } \\
2002 \text { (English) [76] }\end{array}$ & NP & 102 & - & $a=.87-.92$ & ICC $=.65$ & $\begin{array}{l}r=.50-.71 \mathrm{NDI} \\
r=.44-.63 \\
\text { CNFDS }\end{array}$ & $\begin{array}{l}\mathrm{ES}=1.67 \\
\mathrm{SRM}=1.43\end{array}$ & - \\
\hline $\begin{array}{l}\text { Bolton, 2004 } \\
\text { (English) [80] }\end{array}$ & NP & 71 & - & - & - & - & $\begin{array}{l}\mathrm{ES}=1.67 \\
\mathrm{SRM}=1.01\end{array}$ & $\begin{array}{l}\mathrm{RCl}(>1.96)=13 \\
\text { points }\end{array}$ \\
\hline $\begin{array}{l}\text { Gay et al, } 2007 \\
\text { (English) [59] }\end{array}$ & $\begin{array}{l}\text { Chronic NP > } \\
3 \text { months }\end{array}$ & 23 & - & $a=.85-.89$ & - & $\begin{array}{l}r=.77-.80 \mathrm{NDI} \\
r=.37-.62 \\
\text { VAS-P }\end{array}$ & $\begin{array}{l}\mathrm{ES}=1.28 \\
\mathrm{SRM}=1.17\end{array}$ & - \\
\hline $\begin{array}{l}\text { Martel et al, } 2009 \\
\text { (French) [77] }\end{array}$ & Chronic NP & 68 & - & - & $\begin{array}{l}r=.97(95 \% \\
C 195-.98) \\
\text { ICC }=.97(95 \% \\
\text { Cl. } .95-.98)\end{array}$ & $r=.61-.67 \mathrm{NDI}$ & $\begin{array}{l}\mathrm{ES}=.56 \\
\mathrm{SRM}=.61\end{array}$ & $\begin{array}{l}\mathrm{RCl}(>1.96)=4.4 \\
\text { points }\end{array}$ \\
\hline $\begin{array}{l}\text { Soklic et al, } 2012 \\
\text { (German) [78] }\end{array}$ & NP & 102 & - & $a=.79-.82$ & $\begin{array}{l}\mathrm{ICC}=.99(95 \% \\
\mathrm{Cl} .98-.99)\end{array}$ & $\begin{array}{l}r=.68-.76 \mathrm{NDI} \\
r=.69-.80 \\
\text { NPDS }\end{array}$ & $\mathrm{SRM}=.73-1.20$ & - \\
\hline $\begin{array}{l}\text { Geri et al, } 2014 \\
\text { (Italian) [23] }\end{array}$ & $\begin{array}{l}\text { Chronic NP } \\
>3 \text { months }\end{array}$ & 96 & $\begin{array}{l}2 \text { factors: pain \& functioning } \\
(\text { F\#1); anxiety \& depression (F\#2) }\end{array}$ & $\begin{array}{l}\text { Total score: } a=.89 \text { (95 \% } \\
\text { Cl.84-.92) } \\
\text { F\#1: } a=.88(95 \% \text { Cl.83-.92) } \\
\text { F\#2: } a=.90(95 \% \text { Cl.86-.94) }\end{array}$ & - & $\begin{array}{l}r=.67-.70 \\
\text { NPDS } \\
r=.63-.73 \text { NRS }\end{array}$ & - & $\begin{array}{l}\text { MCID }=5.5 \text { points } \\
\text { AUC }=.72 \text { (Sens. } \\
75 \% ; \text { Spec. } 60 \% \text { ) }\end{array}$ \\
\hline $\begin{array}{l}\text { Geri et al, } 2015 \\
\text { (Italian) [79] }\end{array}$ & Chronic NP & 161 & $\begin{array}{l}2 \text { factors: pain \& functioning } \\
\text { (F\#1); anxiety \& depression (F\#2) }\end{array}$ & $\begin{array}{l}\text { PSI }(F \# 1)=.80 \\
\text { PSI }(F \# 2)=.77\end{array}$ & - & - & - & - \\
\hline
\end{tabular}

a Cronbach's alpha, AUC area under the curve, CI confidence interval, CNFDS copenhagen neck functional disability scale, ES effect size, ICC intraclass correlation coefficient, $M C I D$ minimal clinically important difference, $N D I$ neck disability index, NP neck pain, NPDS neck pain and disability scale, NRS numerical rating scale, PSI person separation index, $r$ correlation coefficient, $R C l$ reliable change index, Sens sensibility, Spec specificity, SRM standardized response mean, VAS-P visual analogue scale for pain 
Table 5 Psychometric properties of the core outcome measure index

\begin{tabular}{|c|c|c|c|c|c|c|c|c|}
\hline \multirow{2}{*}{$\begin{array}{l}\text { Authors, year } \\
\text { (language) }\end{array}$} & \multirow[t]{2}{*}{ Population } & \multirow{2}{*}{$\begin{array}{l}\text { Sample } \\
\text { size }\end{array}$} & \multirow[t]{2}{*}{ Dimensionality } & \multirow{2}{*}{$\begin{array}{l}\text { Internal } \\
\text { consistency }\end{array}$} & \multirow[t]{2}{*}{ Reliability } & \multirow[t]{2}{*}{ Validity } & \multicolumn{2}{|l|}{ Responsiveness } \\
\hline & & & & & & & Distribution-based methods & Anchor-based methods \\
\hline $\begin{array}{l}\text { White et al., } 2004 \\
\text { (English) [81] }\end{array}$ & Chronic mechanical NP & 133 & - & - & Single items: ICC $=.64-.99$ & $\begin{array}{l}\text { Pain: } r=.73 \text { VAS-P } \\
\text { Other items: } r=.60 \mathrm{NDI}\end{array}$ & - & - \\
\hline $\begin{array}{l}\text { Kovacs et al, } 2008 \\
\text { (Spanish) [82] }\end{array}$ & $\begin{array}{l}\text { Acute, subacute and } \\
\text { chronic NP }\end{array}$ & 167 & - & $\begin{array}{l}\text { Pain: } a=.73 \\
\text { Disability: } \\
a=.84\end{array}$ & $\begin{array}{l}\text { ICC }=.85 \\
(95 \% \mathrm{Cl} .75-.91)\end{array}$ & $\begin{array}{l}r_{p}=.61 \text { VAS-P } \\
r_{p}=.46 \text { VAS-referred P } \\
r_{p}=.57 \mathrm{CSQ} \\
r_{p}=.69 \mathrm{NDI} \\
r_{p}=.71 \mathrm{NPQ} \\
r_{p}=-.60 \mathrm{SF}-12 \mathrm{PF}\end{array}$ & $\begin{array}{l}\text { Pain: } E S=.79 \\
\text { Disability } E S=.92\end{array}$ & - \\
\hline $\begin{array}{l}\text { Miekisiak et al, } 2014 \\
\text { (Polish) [83] }\end{array}$ & $\begin{array}{l}\text { NP } \\
>4 \text { weeks }\end{array}$ & 123 & 1 factor & - & $\begin{array}{l}\text { ICC }=.88 \\
(95 \% \text { Cl .82-.92) }\end{array}$ & $\mathrm{r}_{\mathrm{s}}=.62 \mathrm{NDI}$ & $\begin{array}{l}\mathrm{MDC}=2 / 10 \text { points } \\
\mathrm{SEM}=.7 / 10 \text { points }\end{array}$ & - \\
\hline $\begin{array}{l}\text { Monticone et al, } \\
2014 \text { (Italian) [22] }\end{array}$ & Chronic NP >3 months & 103 & - & - & $\begin{array}{l}\text { ICC }=.87 \\
(95 \% \mathrm{Cl} .81-.91)\end{array}$ & $\begin{array}{l}\text { Pain: } r_{p}=.45 \text { NRS } \\
\text { Pain: } r_{p}=.48 \text { NPDS } \\
\text { Function: } r_{p}=.49-.55 \text { NPDS } \\
\text { QoL: } r_{p}=-.44 \text { EQ-5D } \\
\text { Disability: } r_{p}=.45-.48 \text { NPDS }\end{array}$ & $\begin{array}{l}\mathrm{MDC}=1.8 / 10 \text { points } \\
\mathrm{SEM}=.65 / 10 \text { points } \\
\text { SRM }=1.23\end{array}$ & $\begin{array}{l}\text { AUC: } .73(.62-.85) \\
(\text { Sens }=.55 ; \text { Spec }=.88)\end{array}$ \\
\hline
\end{tabular}

a Cronbach's alpha, AUC area under the curve, Cl confidence interval, CSQ coping strategies questionnaire, EQ-5d Euroqol 5-dimensions, ES effect size, ICC intraclass correlation coefficient, MDC minimum detectable change, NDI neck disability index, NP neck pain, NPDS neck pain and disability scale, NPQ northwick park questionnaire, NRS numeric rating scale, QoL quality of life, $r$ correlation coefficient, $r_{p}$ Pearson's correlation coefficient, $r_{s}$ Sperman's correlation coefficient, SEM standard error of measurement, Sens sensitivity, SF-12 PF 12-item short-form health survey physical functioning subscale, Spec specificity, SRM standardized response mean, VAS-P visual analogue scale for pain 
Table 6 Psychometric properties of the NeckPix ${ }^{\circledast}$

\begin{tabular}{|c|c|c|c|c|c|c|c|c|}
\hline \multirow{2}{*}{$\begin{array}{l}\text { Authors, year } \\
\text { (language) }\end{array}$} & \multirow[t]{2}{*}{ Population } & \multirow{2}{*}{$\begin{array}{l}\text { Sample } \\
\text { size }\end{array}$} & \multirow[t]{2}{*}{ Dimensionality } & \multirow{2}{*}{$\begin{array}{l}\text { Internal } \\
\text { consistency }\end{array}$} & \multirow[t]{2}{*}{ Reliability } & \multirow[t]{2}{*}{ Validity } & \multicolumn{2}{|l|}{ Responsiveness } \\
\hline & & & & & & & $\begin{array}{l}\text { Distribution-based } \\
\text { methods }\end{array}$ & $\begin{array}{l}\text { Anchor-based } \\
\text { methods }\end{array}$ \\
\hline $\begin{array}{l}\text { Monticone et al, } \\
2014 \text { (Italian) [84] }\end{array}$ & $\begin{array}{l}\text { Chronic NP } \\
\text { (>12 weeks) }\end{array}$ & 118 & 1 factor & $a=.95$ & $\begin{array}{l}\mathrm{ICC}=.98(95 \% \\
\mathrm{Cl} .97-.98)\end{array}$ & $\begin{array}{l}r_{p}=.76 \mathrm{TSK} \\
r_{p}=.58 \mathrm{PCS} \\
r_{p}=.52 \mathrm{NDI} \\
r_{p}=.45 \mathrm{NRS}\end{array}$ & - & - \\
\hline
\end{tabular}

$a$ Cronbach's alpha, $C l$ confidence interval, ICC intraclass correlation coefficient, NP neck pain, NDI neck disability index, NRS numeric rating scale, $P C S$ pain catastrophizing scale, $r_{p}$ Pearson's correlation coefficient, TSK tampa scale for kinesiophobia

Factor analysis revealed either two [71], three [20, $28,38,40,55,67]$, or four factors $[38,65,68]$, but the items constituting each factor were not consistent across studies of comparable quality. The average time to complete the questionnaire was reported to be generally lower than $8 \mathrm{~min}[20,28,65]$.

Internal consistency was high, with Cronbach's alpha for the total score ranging from 0.86 [69] to 0.97 [68]. The ICC values were above 0.75 , but only in a few studies of lower quality [20, 28, 32, 38, 73] did they exceed the minimum required value of 0.90 .

The NPDS showed a strong correlation with concurrent scales such as the NDI $[28,32,62,73,74]$ and the Northwick Park Questionnaire (NPQ) [28], moderate to strong correlations with VAS pain [28, 31, 38, 40, 69, 71], and a weak to moderate correlation with SF-36 [20, $32,38,71]$. The NPDS demonstrated good face validity, being able to discriminate $(p<.01)$ patients with neck pain from healthy subjects or subjects with low back and leg pain [65]. Content validity was confirmed by the high rate of answers to all items, while the most common missing items concerned driving, reading, and medication $[32,40,70,74]$. There were no floor or ceiling effects found [28, 29, 32, 40, 63, 72, 75].

The ES and SRM values reported varied widely across studies. Because these indices are based on standard deviations, the differences observed may be due to the sample size or patient selection of the studies. Similarly, the different methods adopted to calculate the MDC across studies led to very different results in the studies of poor quality, ranging from 3 [72] to 31.7 points [64]. The MCID was close to 10 points both for the Italian version in a study of excellent quality (AUC 0.91; sensitivity 0.93; specificity 0.83) [24] and for the Dutch version in a low quality study (11.5 points; AUC 0.75 ; sensibility 0.74; specificity 0.70) [64].

\section{Neck Bournemouth questionnaire}

The NBQ is a self-report questionnaire developed to measure neck pain according to the biopsychosocial model [76]. It consists of 7 items rated on a NRS from 0 to 10 (where 0 means 'much better', 5 'no change', and 10 'much worse') for a total score range 0-70, with higher scores reflecting more severity. The NBQ has been translated into several languages, including French [77], German [78], and Italian [23] (Table 4).

Factor analysis was conducted on the Italian version in a good quality study, and revealed a model composed of two different subscales dealing with pain \& functioning (factor 1 , items \#1, \#2, \#3, \#6, and \#7, explaining $56.6 \%$ of the variance), and anxiety \& depression (factor 2, item \#4 and \#5, explaining $12.6 \%$ of the variance) [23]. Cronbach's alpha for the total score ranged from 0.79 [78] to 0.92 [76], indicating a high interrelatedness of the items with a possible tendency to redundancy. The internal consistency of the two subscales revealed a similar pattern [23]. Confirmatory factor analysis indicated item \#7 as unnecessary in factor 1, while for factor 2 the high redundancy could be attributable to the overlapping of feelings like anxiety and depression [23]. A recent Rasch Italian study [79] confirmed the presence of two factors. After removal of item \#7, the first factor (pain \& functioning) fitted the Rasch model, while the second factor (anxiety \& depression) fitted the model without modification. The time needed to complete the questionnaire is less than 5 min [23, 76]. Test-retest reliability ranged from moderate [76] to excellent $[77,78]$.

The NBQ showed a moderate to strong correlation with most existing questionnaires, such as NDI [59, 76-78], NPDS [23, 78], and the Copenhagen Neck Functional Disability Scale [76], but a weak to moderate correlation with VAS pain [59]. A large portion of patients judged the NBQ as relevant to their health problem (78.7\%) or as relevant for other people with neck pain (87.9 \%) [79], confirming the face validity of the questionnaire. A floor effect (19.4\% of patients attained the lowest score) was observed in the anxiety and depression factor's score after treatment [79].

The NBQ was considered a sensitive outcome measure able to depict moderate-to-large change in groups of patients with NSNP. The MCID was estimated using both ROC and Reliable Change Index methods. Two studies of fair to good quality reported similar findings, ranging from 4.4 [77] to 5.5 points [23], but higher raw change scores of 13 points or more (and percentage change scores of $36 \%$ or more) were also reported in a study of poor quality as giving the best balance between sensitivity and specificity in detecting clinically improved 
patients [80]. The MDC of the questionnaire has never been calculated.

\section{Core outcome measures index for neck pain}

This questionnaire was adapted with some minor changes from the existing low back pain version. It contains seven items pertaining to five domains: severity of pain, function, symptom-specific well-being, quality of life, and disability (social and work). Items refer to how the subject felt in the last week, except for those regarding disability which refer to the last month. Pain items use a $0-10 \mathrm{~cm}$ VAS and the higher of the two scores is used to represent pain. The other items use a 5-point Likert-type scale. The COMI score is calculated by averaging the values for each domain (with higher scores indicating a worse status) into a 0-5 score $[81,82]$ or more recently - after re-scoring them on a 0-10 scale $[22,83]$. The COMI has been translated into Spanish [82], Polish [83], and Italian [22] (Table 5). The time required to complete the questionnaire is less than $3 \mathrm{~min}$ and the acceptability was found to be good, as shown by the absence of problems in comprehension or of missing or multiple answers [22].

Factorial analysis was performed only on the Polish version in a study of excellent methodological quality [83], and a single factor explaining $61.6 \%$ of the variation in score was identified. Internal consistency was measured only for the pain and disability subscales with acceptable values in a poor quality study [82], and the test-retest reliability of the total score was almost high $[23,82]$. The COMI total score was found to be consistent with the external criterion for disability (values increased as patients' self-perception of disability increased), but not with that for pain [82]. The COMI showed a lower correlation than other questionnaires (e.g. NDI and NPQ) with measures of pain or disability. The Italian [23] and Polish [83] versions showed also some floor and ceiling effects.

The COMI was found to be poorly sensitive to worsening of both pain and disability; it reflected improvement in pain for patients who denied any change, and it magnified the amount of improvement for pain and, especially, for disability [82]. MDC values were about $2 / 10$ points for both the Italian [23] and Polish versions [83] in good quality studies. The ROC analysis was carried out on the COMI change scores in a study of poor methodological quality, revealing a significant ability to discriminate poor from good patients, with the cut-off set at two points [23].

\section{NeckPix@}

This measure [84] was recently developed in Italian to assess activity-related kinesiophobia in outpatients with chronic NSNP (Table 6). It consists of ten images that represent everyday activities involving the neck. The patient rates from 0 to $10(0=$ no fear, $10=$ greatest fear $)$ the fear of feeling pain in the neck when doing the activity represented in each image. The total score ranges from 0 to 100. The scale requires a mean time of $2 \mathrm{~min}$ to complete.

An excellent methodological quality exploratory factor analysis revealed a one-factor structure [84]. The internal consistency and reliability were excellent, and good correlations were found with the Tampa Scale of Kinesiophobia and the Pain Catastrophizing Scale. No floor or ceiling effects were observed.

\section{Discussion}

Four instruments measuring function and disability, and one measuring activity-related fear of movement, are now available for assessing Italian people with nonspecific neck pain. In 2011, a systematic review [5] of translated versions of neck-specific questionnaires was able to identify only one instrument. Overall, the available information on measurement properties of the Italian versions of PROMs for NSNP are good, despite the poor methodological quality of most translations.

\section{Psychometric properties}

Among the instruments considered in this review, the NDI is the one that has been most widely studied. It is the only instrument having all the measurement properties validated and with positive findings $[4,5]$. However, important issues regarding dimensionality and responsiveness emerged. Factor analysis raised uncertainty about the presence of a single construct, which was definitively rejected by RA [44, 46, 56-58]. Unidimensionality could be achieved by removing from 1 [44] to 5 [58] of the 10 original items. While item \#5 (headache) was a common misfitting item (headache may not be a common symptom experienced by all neck pain patients, and therefore not sensitive to change) [57], there was no consistency between studies on which items exactly should be removed. The NDI showed also a large floor effect [56]. As a result, the NDI may be inadequate to assess patients with moderate to high functioning, and it may not be sensitive to changes in patients' functioning over time. Problems with responsiveness were also related to the large variability of measurement error [30,60], and a poor raw score to measure correlation was found [56]. Before adopting the NDI as the instrument of first choice and determining a range for MCID, the dimensionality, reliability and measurement error of this questionnaire needs to be carefully assessed.

The NPDS was the first instrument translated into Italian, and its measurement properties have been extensively examined. However, agreement on its dimensionality is still lacking. The developers originally described a 
4-factor structure, but the Italian validation study extracted only three factors. The high variability among studies precludes any confident judgement about the factorial structure and content of the scale. This raises the need for RA to test its dimensionality and metrics before it can be recommended to interpret clinical changes in individual patients. Future studies should also carefully estimate the measurement error, to verify that it does not exceed the MCID.

The NBQ demonstrated acceptable psychometric properties when tested with CTT methods. The results of both factor analysis and RA revealed a robust 2 -factor structure [23, 79], and a refined version with removal of item \#7 was proposed [79]. This implies that two independent subscales should be used in place of a total composite score. Subscale 1 was intended to measure neck-related disability (similar to that of the NDI) and was better suited to assess the health status of patients with chronic NSNP in research settings [79]. Subscale 2, dealing with anxiety \& depression, should be used with caution given the presence of only two items. To avoid biased conclusions about treatment effectiveness, it was recommended to use the Rasch-conversion tables provided for each subscale of the Italian version [79]. The responsiveness should be also re-assessed taking into consideration the deletion of item \#7 from subscale 1. After that, the NBQ could be considered a valid instrument to measure quality of life in people suffering from NSNP.

The COMI has been less extensively studied than the instruments above, and some problems regarding the sensitivity to change have emerged. The exploratory factor analysis showed a mono-factorial structure, but the paucity of information about the dimensionality of this scale warrants further investigation with RA. Inconsistencies between studies also emerged in this review, in particular concerning the methods used to calculate the total score, the classification of items, and the scoring categories of some items. This could lead to misunderstandings when comparing results across studies.

The NeckPix@ - recently developed in Italy - showed a robust factorial structure and good reliability and validity. However, no information about its responsiveness was provided by the developers. It constitutes an innovative and promising measure of activity-related kinesiophobia, but before it can be recommended as an outcome measure for clinical and research purposes, this instrument needs to undergo further research to confirm its measurement properties and clarify how to interpret the results.

\section{Clinical utility}

Among the PROMs with comparable validity, reliability and responsiveness, the choice of which measurement tool to use should be made only after a careful evaluation of the clinical utility, and depends on what type of intervention is planned and what the anticipated response is. The clinical utility of a measure relates to its ease and efficiency of use, and to the relevance and meaningfulness of the information that it provides [85]. No substantial differences in core elements such as ease of use, time taken to administer, training and qualification of clinicians required, format (acceptability), and cost were observed between the instruments evaluated in this study. On the other hand, differences emerged as to their content (i.e. which domains the PROMs are intended to measure), and this may be of greater interest to clinicians who need to make a precise assessment of specific aspects that affect patients with NSNP. The content of NeckPix@ is appropriate for evaluating activityrelated fear of movement, while the other four instruments are aimed at measuring mainly function and disability, and could be classified using the ICF [86] framework. The ICF identifies two different relevant domains that should be addressed: 1) Functioning, Disability and Health, which includes: i) Body Functions, ii) Body Structures, iii) Activity and Participation; and 2) Contextual Factors, that include: i) Environmental Factors, and ii) Personal Factors [87]. As there is currently no core set of domains for neck pain assessment, the patient's own experience has been used to classify their functional problems and these have been linked to the ICF. Problems with functioning belonging to the Activities and Participation component (such as computer work, driving, maintaining a body position, lifting and carrying objects) were the most frequently reported [88]. However, patients with neck problems reported also a higher proportion of body function impairments (such as sleep disturbance, functional problems with mobility of joint functions) than patients with musculoskeletal pain in other body regions [87]. That indicates a multidimensionality of their functional problems, and requires an in-depth assessment.

For the purposes of the present study, PROMs were linked to the ICF framework within the components described above. However, coding questionnaires is not always straightforward: items of each instrument could be linked across more than one category, or may not be classified at all. The NDI had four items (40 \%) categorized as body functions, and six items classified as activity and participation (60\%); the NPDS contained 11 items ( $55 \%$ ) classified as body functions, eight in the activity and participation category (40\%), and 1 (5\%) pertaining to environmental factors; the NBQ had three items (43\%) classified as body functions and 3 (43\%) as activity and participation (one item could not be classified into the ICF categories); the COMI had two items (33 \%) classified as body functions and $4(67 \%)$ as 
activity and participation. All four instruments showed a well-balanced distribution of items across the body functions and activity and participation components, although in different ratios and with a different ICF category coverage. For example, the NPDS is the only one that assesses contextual factors such as drug use.

NSNP is a complex, multidimensional experience and it is imperative that PROMs assess and reflect this accurately, in order to be useful in both the clinical and research settings. Multimodal interventions may be more effectively measured by a scale that can be demonstrated to measure a variety of factors that contribute to neck pain and related disability. However, the disadvantage of using multidimensional scales is that interpreting the meaning of the overall score and determining the attribution of changes becomes more difficult.

\section{Limitations}

The search was restricted to studies published in English and Italian. However, as the aim of this review was to identify the PROMs validated in Italian, the likelihood of further relevant articles published in different languages was very low. It should also be noted that this study examined those PROMs aimed to evaluate patients with NSNP only, so data extracted from other samples (e.g. in patients with whiplash or after neck surgery) were excluded. The risk of bias of the studies included in this review was not assessed, as most information was considered from studies at low risk of bias.

\section{Conclusions}

In the last 5 years, four instruments (NDI, NPDS, NBQ, and COMI) have been translated into Italian language with the aim to measure function and disability and one (NeckPix@) to measure activity-related fear of movement. The most widespread PROM is the NDI, but important issues about its dimensionality and responsiveness emerged, especially in patients with moderate to high functioning. The NPDS has also been extensively investigated, but the agreement on its dimensionality is still lacking. The NBQ has demonstrated good psychometric properties, especially in the Italian version. If they are confirmed by further studies, this scale could be considered as a comprehensive tool for measuring pain \& functioning, and anxiety \& depression in patients with NSNP.

\section{Appendix}

\section{Search strategies for each investigated database MEDLINE}

("Psychometrics"[Mesh] OR "Outcome Assessment (Health Care)"[Mesh] OR "Validation Studies as Topic"[Mesh] OR "Validation Studies"[Publication Type] OR "Questionnaires"[Mesh] OR "Evaluation Studies"[Publication Type] OR “Translations" [Mesh] OR "Translating"[Mesh] OR "Cross-
Cultural Comparison"[Mesh] OR "Reproducibility of Results"[Mesh] OR valid* OR accuracy OR reliability OR agreement OR reproducibility OR "sensitivity to change" OR responsiveness OR "minimal detectable change" OR "minimal clinical" important change" OR "minimal clinical" important difference" OR "minimal important change" OR "floor effect" OR "ceiling effect" OR "factor analysis" OR translation OR rasch OR psychometrics OR version) AND ("Neck Pain"[Mesh] OR "neck pain" OR "cervical pain").

\section{EMBASE}

('outcome assessment'/exp OR 'outcome assessment' OR 'validation studies'/exp OR 'validation studies' OR 'questionnaires'/exp OR questionnaires OR 'evaluation studies'/ exp OR 'evaluation studies' OR 'translati"' OR 'cross-cultural validation' OR valid* OR 'accuracy'/exp OR accuracy OR 'reliability'/exp OR reliability OR agreement OR 'reproducibility'/exp OR reproducibility OR 'sensitivity to change' OR responsiveness OR 'minimal detectable change' OR 'minimal clinical" important change' OR 'minimal clinical" important difference' OR 'minimal important change' OR 'floor effect' OR 'ceiling effect' OR 'factor analysis'/exp OR 'factor analysis' OR translation OR rasch OR 'psychometrics'/exp OR psychometrics OR version) AND ('neck pain'/ exp OR 'neck pain' OR 'cervical pain').

\section{CINHAL}

("Outcome Assessment" OR "Validation Studies" OR Questionnaires OR "Evaluation Studies" OR Translati" OR "Cross-Cultural Validation" OR valid" OR accuracy OR reliability OR agreement OR reproducibility OR "sensitivity to change" OR responsiveness OR "minimal detectable change" OR "minimal clinical" important change" OR "minimal clinical" important difference" OR "minimal important change" OR "floor effect" OR "ceiling effect" OR "factor analysis" OR translation OR rasch OR psychometrics OR version) AND ("Neck Pain" OR "cervical pain").

\section{Scopus, Web of Science, Cochrane Library}

(Questionnaires OR validity OR accuracy OR reliability OR agreement OR reproducibility OR "sensitivity to change" OR responsiveness OR "factor analysis" OR translation OR rasch OR psychometrics OR version) AND ("Neck Pain" OR "cervical pain").

\footnotetext{
Abbreviations

AUC, area under curve; COMI, core outcome measures index; COSMIN, COnsensus-based Standards for the selection of health Measurement INstruments; CTT, classical test theory; ES, effect size; GRI, Guyatt's responsiveness index; ICC, intraclass coefficient correlation; ICF, international classification of functioning disability and health; MCID, minimal clinically important difference; $M D C$, minimum detectable change; $N B Q$, neck Bournemouth Questionnaire; NDI, neck disability index; NPDS, neck pain and disability scale; NPDS, neck pain and disability scale; NPQ, northwick park questionnaire; NRS, numeric rating scale; NSNP, non-specific neck pain; PROM, patientreported outcome measure; RA, Rasch analysis; ROC, receiver operating
} 
characteristic; SF-36, medical outcomes study 36-item short-form health survey; SRM, standardized response mean; VAS, visual analogue scale

\section{Acknowledgements}

Not applicable.

\section{Funding}

No funding was received for this manuscript.

\section{Authors' contributions}

LP participated in study design, data extraction, assessment of the methodological quality of the studies included, analysis and interpretation of results; FB participated in study design, inclusion of articles, analysis and interpretation of results; DDF has participated in study design, inclusion of articles, analysis and interpretation of results; MM participated in study design, inclusion of articles, analysis and interpretation of results; SV participated in study design, data extraction, assessment of the methodological quality of the studies included in the analysis and interpretation of results. All the authors were involved in drafting the manuscript or revising it critically for important intellectual content, and they have given their final approval to the version to be published.

\section{Competing interests}

The authors declare that they have no competing interests.

\section{Consent for publication}

Not applicable.

\section{Ethics approval and consent to participate}

Not applicable.

\section{Author details}

${ }^{1}$ Program in Advanced Sciences and Technologies in Rehabilitation and Sports Medicine, Tor Vergata University, Rome, Italy. ${ }^{2}$ Department of Clinical Sciences and Translational Medicine, Tor Vergata University, Rome, Italy. ${ }^{3}$ Department of Rehabilitation, Rome American Hospital, Rome, Italy. ${ }^{4}$ Department of Neuroscience, Rehabilitation, Ophthalmology, Genetics, Maternal and Child Health, University of Genoa - Campus of Savona, Savona, Italy. ${ }^{5}$ Laboratory of Ergonomics and Musculoskeletal Disorders Assessment, Division of Physical Medicine and Rehabilitation, Salvatore Maugeri

Foundation, Scientific Institute of Veruno, IRCCS, Veruno, NO, Italy.

\section{Received: 23 February 2016 Accepted: 13 July 2016}

\section{Published online: 22 July 2016}

\section{References}

1. Binder A. Neck pain. Clin Evid. 2006;15:1654-75.

2. Haldeman S, Carroll L, Cassidy JD. Findings from the bone and joint decade 2000 to 2010 task force on neck pain and its associated disorders. J Occup Environ Med. 2010. doi:10.1097/JOM.0b013e3181d44f3b.

3. Reeve BB, Wyrwich KW, Wu AW, Velikova G, Terwee GB, et al. ISOQOL recommends minimum standards for patient-reported outcome measures used in patient-centered outcomes and comparative effectiveness research. Qual Life Res. 2013. doi:10.1007/s11136-012-0344-y.

4. Schellingerhout JM, Verhagen AP, Heymans MW, Koes BW, de Vet HC, Terwee CB. Measurement properties of disease-specific questionnaires in patients with neck pain: a systematic review. Qual Life Res. 2012. doi:10.1007/s11136-011-9965-9.

5. Schellingerhout JM, Heymans MW, Verhagen AP, de Vet HC, Koes BW, Terwee CB. Measurement properties of translated versions of neck-specific questionnaires: a systematic review. BMC Med Res Methodol. 2011. doi:10.1186/1471-2288-11-87.

6. Mokkink LB, Terwee CB, Patrick DL, Alonso J, Stratford PW, Knol DL, Bouter LM, de Vet HC. The COSMIN study reached international consensus on taxonomy, terminology, and definitions of measurement properties for health-related patient-reported outcomes. J Clin Epidemiol. 2010. doi:10.1016/j.jclinepi.2010. 02.006 .

7. Terwee CB, Bot SD, de Boer MR, van der Windt DA, Knol DL, et al. Quality criteria were proposed for measurement properties of health status questionnaires. J Clin Epidemiol. 2007;60:34-42.
8. Portney LG, Watkins MP. Foundations of clinical research. Applications to practice. East Norwalk: Appleton \& Lange; 1993.

9. Campbell DT, Fiske DW. Convergent and discriminant validation by the multitrait-multimethod matrix. Psychol Bull. 1959:56:81-105.

10. Munro B. Statistical methods for health care research. Philadelphia: JB Lippincott; 2000.

11. Franchignoni F, Vercelli S, Giordano A, Sartorio F, Bravini E, Ferriero G. Minimal clinically important difference of the Disabilities of the Arm, Shoulder and Hand outcome measure (DASH) and its shortened version (QuickDASH). J Orthop Sports Phys Ther. 2014. doi:10.2519/jospt.2014.4893.

12. Revicki D, Hays RD, Cella D, Sloan J. Recommended methods for determining responsiveness and minimally important differences for patient-reported outcomes. J Clin Epidemiol. 2008. doi:10.1016/j.jclinepi.2007.03.012.

13. Turner D, Schünemann HJ, Griffith LE, Beaton DE, Griffiths $A M$, Critch JN, Guyatt $\mathrm{GH}$. The minimal detectable change cannot reliably replace the minimal important difference. J Clin Epidemiol. 2010. doi:10.1016/j.jclinepi.2009.01.024.

14. Terwee CB, Roorda LD, Dekker J, Bierma-Zeinstra SM, Peat G, et al. Mind the MIC: large variation among populations and methods. J Clin Epidemiol. 2010. doi:10.1016/j.jclinepi.2009.08.010.

15. Wright AA, Cook CE, Baxter GD, Dockerty JD, Abbott JH. A comparison of 3 methodological approaches to defining major clinically important improvement of 4 performance measures in patients with hip osteoarthritis. J Orthop Sports Phys Ther. 2011. doi:10.2519/jospt.2011.3515.

16. Tesio L. Measuring behaviours and perceptions: Rasch analysis as a tool for rehabilitation research. J Rehabil Med. 2003;35:105-15.

17. Bond TG, Fox CM. Applying the Rasch model: fundamental measurement in the human sciences. Mahwah: Lawrence Erlbaum Associates; 2001.

18. Conrad KJ, Smith Jr EV. International conference on objective measurement: applications of Rasch analysis in health care. Med Care. 2004:42 Suppl 1:1-6.

19. Linacre JM. Rasch power analysis: size vs. significance: infit and outfit meansquare and standardized chi-square fit statistic. Rasch Meas Trans. 2003;17:918.

20. Monticone M, Baiardi P, Nido N, Righini C, Tomba A, Giovanazzi E. Development of the Italian version of the Neck Pain and Disability Scale, NPDS-l: cross-cultural adaptation, reliability, and validity. Spine (Phila Pa 1976). 2008. doi:10.1097/BRS. Ob013e318175c2bo.

21. Monticone M, Ferrante S, Vernon H, Rocca B, Dal Farra F, Foti C. Development of the Italian version of the Neck Disability Index: cross-cultural adaptation, factor analysis, reliability, validity, and sensitivity to change. Spine (Phila Pa 1976). 2012. doi:10.1097/BRS.0b013e3182579795.

22. Monticone M, Ferrante S, Maggioni S, Grenat G, Checchia GA, et al. Reliability, validity and responsiveness of the cross-culturally adapted Italian version of the Core Outcome Measures Index (COMI) for the neck. Eur Spine J. 2014. doi:10.1007/s00586-013-3092-y.

23. Geri T, Signori A, Gianola S, Rossettini G, Grenat G, Checchia G, Testa M. Cross-cultural adaptation and validation of the Neck Bournemouth Questionnaire in the Italian population. Qual Life Res. 2014. doi:10.1007/ s11136-014-0806-5.

24. Monticone M, Ambrosini E, Vernon H, Brunati R, Rocca B, Foti C, Ferrante S. Responsiveness and minimal important changes for the Neck Disability Index and the Neck Pain Disability Scale in Italian subjects with chronic neck pain. Eur Spine J. 2015 doi:10.1007/s00586-015-3785-5.

25. Vernon H, Mior S. The Neck Disability Index: a study of reliability and validity. J Manipulative Physiol Ther. 1991:14:409-15.

26. Chok B, Gomez E. The reliability and application of the Neck Disability Index in physiotherapy. Physiotherapy Singapore. 2000;3:16-9.

27. Ackelman BH, Lindgren U. Validity and reliability of a modified version of the Neck Disability Index. J Rehabil Med. 2002;34:284-7.

28. Wlodyka-Demaille S, Poiraudeau S, Catanzariti JF, Rannou F, Fermanian J, Revel M. French translation and validation of 3 functional disability scales for neck pain. Arch Phys Med Rehabil. 2002;83:376-82.

29. Lee H, Nicholson LL, Adams RD, Maher CG, Halaki M, Bae SS. Development and psychometric testing of Korean language versions of 4 neck pain and disability questionnaires. Spine (Phila Pa 1976). 2006;31:1841-5.

30. Vos CJ, Verhagen AP, Koes BW. Reliability and responsiveness of the Dutch version of the Neck Disability Index in patients with acute neck pain in general practice. Eur Spine J. 2006;15:1729-36.

31. Kose G, Hepguler S, Atamaz F, Oder G. A comparison of four disability scales for Turkish patients with neck pain. J Rehabil Med. 2007:39:358-62.

32. Mousavi SJ, Parnianpour M, Montazeri A, Mehdian H, Karimi A, et al. Translation and validation study of the Iranian versions of the Neck Disability Index and the Neck Pain and Disability Scale. Spine. 2007;32:E825-31. 
33. Trouli MN, Vernon HT, Kakavelakis KN, Antonopoulou MD, Paganas AN, Lionis CD. Translation of the Neck Disability Index and validation of the Greek version in a sample of neck pain patients. BMC Musculoskelet Disord. 2008. doi:10.1186/1471-2474-9-106.

34. Andrade Ortega JA, Delgado Martinez AD, Ruiz RA. Validation of the Spanish version of the Neck Disability Index. Spine (Phila Pa 1976). 2010. doi:10.1097/BRS.0b013e3181afea5d.

35. Odole AC, Adegoke BO, Akomas NC. Validity and test re-test reliability of the Neck Disability Index in the Nigerian clinical setting. Afr J Med Med Sci. 2011;40:135-8.

36. Telci EA, Karaduman A, Yakut Y, Aras B, Simsek IE, Yagli N. The cultural adaptation, reliability, and validity of neck disability index in patients with neck pain: a Turkish version study. Spine (Phila Pa 1976). 2009. doi:10.1097/BRS.0b013e3181ac9055

37. Salo P, Ylinen J, Kautiainen H, Arkela-Kautiainen M, Hakkinen A. Reliability and validity of the Finnish version of the Neck Disability Index and the modified neck pain and disability scale. Spine (Phila Pa 1976). 2010. doi:10.1097/BRS.0b013e3181b327ff.

38. Wu S, Ma C, Mai M, Li G. Translation and validation study of Chinese versions of the Neck Disability Index and the neck pain and disability scale. Spine (Phila Pa 1976). 2010. doi:10.1097/BRS.0b013e3181c6ea1b.

39. Shakil H, Khan SA, Thakur PC. Test retest reliability and validity of Hindi version of Neck Disability Index in patients with neck pain. Indian J Physiother Occup Ther. 2011:5:167-9.

40. Uthaikhup S, Paungmali A, Pirunsan U. Validation of Thai versions of the Neck Disability Index and neck pain and disability scale in patients with neck pain. Spine (Phila Pa 1976). 2011. doi:10.1097/BRS.0b013e31820e68ac.

41. Kesiktas N, Ozcan E, Vernon H. Clinimetric properties of the Turkish translation of a modified Neck Disability Index. BMC Musculoskelet Disord. 2012. doi:10.1186/1471-2474-13-25.

42. Luksanapruksa P, Wathana-apisit T, Wanasinthop S, Sanpakit S, Chavasiri C. Reliability and validity study of a Thai version of the Neck Disability Index in patients with neck pain. J Med Assoc Thai. 2012;95:681-8.

43. Nakamaru K, Vernon H, Aizawa J, Koyama T, Nitta O. Crosscultural adaptation, reliability, and validity of the Japanese version of the Neck Disability Index. Spine (Phila Pa 1976). 2012. doi:10.1097/BRS.0b013e318267f7f5.

44. Ailliet $\mathrm{L}$, Knol DL, Rubinstein SM, De Vet HCW, Van Tulder MW, Terwee CB. Definition of the construct to be measured is a prerequisite for the assessment of validity. The Neck Disability Index as an example. J Clin Epidemiol. 2013. doi:10.1016/j.jclinepi.2013.02.005.

45. Guzy G, Vernon H, Polczyk R, Szpitalak M. Psychometric validation of the authorized Polish version of the Neck Disability Index. Disabil Rehabil. 2013. doi:10.3109/09638288.2013.771706.

46. Johansen JB, Andelic N, Bakke E, Holter EB, Mengshoel AM, Roe C. Measurement properties of the Norwegian version of the Neck Disability Index in chronic neck pain. Spine (Phila Pa 1976). 2013;38(10):851-6.

47. Shaheen AA, Omar MT, Vernon H. Cross-cultural adaptation, reliability, and validity of the Arabic version of Neck Disability Index in patients with neck pain. Spine (Phila Pa 1976). 2013. doi:10.1097/BRS.0b013e31828b2d09.

48. Cramer H, Lauche R, Langhorst J, Dobos GJ, Michalsen A. Validation of the German version of the Neck Disability Index (NDI). BMC Musculoskelet Disord. 2014. doi:10.1186/1471-2474-15-91.

49. Cruz EB, Fernandes R, Carnide F, Domingues L, Pereira M, Duarte S. Cross-cultural adaptation and validation of the Neck Disability Index to European Portuguese language. Spine (Phila Pa 1976). 2015. doi:10.1097/BRS.0000000000000692.

50. Joseph SD, Bellare B, Vernon H. Cultural adaptation, reliability, and validity of Neck Disability Index in Indian rural population: a Marathi version study. Spine (Phila Pa 1976). 2015. doi:10.1097/BRS.0000000000000681.

51. Bakhtadze MA, Vernon H, Zakharova OB, Kuzminov KO, Bolotov DA. The Neck Disability Index-Russian language version (NDI-RU): a study of validity and reliability. Spine (Phila Pa 1976). 2015. doi:10.1097/BRS.0000000000000880.

52. Swanenburg J, Humphreys K, Langenfeld A, Brunner F, Wirth B. Validity and reliability of a German version of the Neck Disability Index (NDI-G). Man Ther. 2014. doi:10.1016/j.math.2013.07.004

53. Hains F, Waalen J, Mior S. Psychometric properties of the Neck Disability Index. J Manipulative Physiol Ther. 1998;21:75-80.

54. Stratford PW, Riddle DL, Binkley JM. Using the Neck Disability Index to make decisions concerning individual patients. Physiother Can. 1999;2:107-12.

55. Pickering PM, Osmotherly PG, Attia JR, McElduff P. An examination of outcome measures for pain and dysfunction in the cervical spine: a factor analysis. Spine (Phila Pa 1976). 2011. doi:10.1097/BRS.0b013e3181d762da.
56. Hung M, Cheng C, Hon SD, Franklin JD, Lawrence BD, et al. Challenging the norm: further psychometric investigation of the Neck Disability Index. Spine J. 2015. doi:10.1016/j.spinee.2014.03.027.

57. van der Velde G, Beaton D, Hogg-Johnston S, Hurwitz E, Tennant A. Rasch analysis provides new insights into the measurement properties of the Neck Disability Index. Arthritis Rheum. 2009. doi:10.1002/art.24399.

58. Walton DM, MacDermid JC. A brief 5-item version of the Neck Disability Index shows good psychometric properties. Health Qual Life Outcomes. 2013. doi:10.1186/1477-7525-11-108.

59. Gay RE, Madson TJ, Cieslak KR. Comparison of the Neck Disability Index and the Neck Bournemouth Questionnaire in a sample of patients with chronic uncomplicated neck pain. J Manipulative Physiol Ther. 2007;30:259-62.

60. Cleland JA, Childs JD, Whitman JM. Psychometric properties of the Neck Disability Index and Numeric Pain Rating Scale in patients with mechanical neck pain. Arch Phys Med Rehabil. 2008. doi:10.1016/j.apmr.2007.08.126.

61. Young BA, Walker MJ, Strunce JB, Boyles RE, Whitman JM, Childs JD. Responsiveness of the Neck Disability Index in patients with mechanical neck disorders. Spine J. 2009. doi:10.1016/j.spinee.2009.06.002.

62. Jorritsma W, De Vries GE, Dijkstra PU, Geertzen JHB, Reneman MF. Neck Pain and Disability Scale and Neck Disability Index: validity of Dutch language versions. Eur Spine J. 2012a. doi:10.1007/s00586-011-1920-5.

63. Pool JJ, Ostelo RW, Hoving JL, Bouter LM, de Vet HC. Minimal clinically important change of the Neck Disability Index and the Numerical Rating Scale for patients with neck pain. Spine (Phila Pa 1976). 2007;32:3047-51.

64. Jorritsma W, Dijkstra PU, De Vries GE, Geertzen JHB, Reneman MF. Detecting relevant changes and responsiveness of Neck Pain and Disability Scale and Neck Disability Index. Eur Spine J. 2012b. doi:10.1007/s00586-012-2407-8.

65. Wheeler AH, Goolkasian P, Baird AC, Darden 2nd BV. Development of the Neck Pain and Disability Scale. Item analysis, face, and criterion-related validity. Spine (Phila Pa 1976). 1999;24:1290-4

66. Million R, Nilsen KH, Jayson MI, Baker RD. Evaluation of low back pain and assessment of lumbar corsets with and without back supports. Ann Rheum Dis. 1981;40:449-54.

67. Scherer M, Blozik E, Himmel W, Laptinskaya D, Kochen MM, HerrmannLingen C. Psychometric properties of a German version of the Neck Pain and Disability Scale. Eur Spine J. 2008. doi:10.1007/s00586-008-0677-y.

68. Chen Z, Zhao Y, Wang C, Li M, Zhu X. An adapted Chinese version of Neck Pain and Disability Scale: validity and reliability. Spine (Phila Pa 1976). 2011. doi:10.1097/BRS.0b013e318209990b.

69. Bicer A, Yazici A, Camdeviren H, Erdogan C. Assessment of pain and disability in patients with chronic neck pain: reliability and construct validity of the Turkish version of the Neck Pain and Disability Scale. Disabil Rehabil. 2004:26:959-62

70. Jorritsma W, De Vries GE, Geertzen JHB, Dijkstra PU, Reneman MF. Neck Pain and Disability Scale and the Neck Disability Index: reproducibility of the Dutch language versions. Eur Spine J. 2010. doi:10.1007/s00586-010-1406-X.

71. Ono R, Otani K, Takegami M, Suzukamo Y, Goolkasian P, et al. Reliability, validity, and responsiveness of the Japanese version of the Neck Pain and Disability Scale. J Orthop Sci. 2011. doi:10.1007/s00776-011-0053-3.

72. Blozik E, Himmel W, Kochen MM, Herrmann-Lingen C, Scherer M. Sensitivity to change of the Neck Pain and Disability Scale. Eur Spine J. 2011. doi:10.1007/s00586-010-1545-0.

73. Goolkasian P, Wheeler AH, Gretz SS. The Neck Pain and Disability Scale: testretest reliability and construct validity. Clin J Pain. 2002;18:245-50.

74. Chan Ci En M, Clair DA, Edmondston SJ. Validity of the Neck Disability Index and Neck Pain and Disability Scale for measuring disability associated with chronic, non-traumatic neck pain. Man Ther. 2009. doi:10.1016/j. math.2008.07.005

75. Wlodyka-Demaille S, Poiraudeau S, Catanzariti JF, Rannou F, Fermanian J, Revel M. The ability to change of three questionnaires for neck pain. Joint Bone Spine. 2004;71:317-26.

76. Bolton JE, Humphreys BK. The Bournemouth questionnaire: a short-form comprehensive outcome measure. II. Psychometric properties in neck pain patients. J Manipulative Physiol Ther. 2002;25:141-8.

77. Martel J, Dugas C, Lafond D, Descarreaux M. Validation of the French version of the Bournemouth questionnaire. J Can Chiropr Assoc. 2009;53:102-20.

78. Soklic M, Peterson C, Humphreys BK. Translation and validation of the German version of the Bournemouth questionnaire for neck pain. Chiropr Man Therap. 2012. doi:10.1186/2045-709X-20-2.

79. Geri T, Piscitelli D, Meroni R, Bonetti F, Giovannico G, Traversi R, Testa M. Rasch analysis of the Neck Bournemouth questionnaire to measure 
disability related to chronic neck pain. J Rehabil Med. 2015. doi:10.2340/16501977-2001.

80. Bolton JE. Sensitivity and specificity of outcome measures in patients with neck pain: detecting clinically significant improvement. Spine (Phila Pa 1976). 2004;29(21):2410-7.

81. White P, Lewith G, Prescott P. The core outcomes for neck pain: validation of a new outcome measure. Spine (Phila Pa 1976). 2004;29:1923-30.

82. Kovacs FM, Bagò J, Royuela A, Seco J, Gimenez S, et al. Psychometric characteristics of the Spanish version of instruments to measure neck pain disability. BMC Musculoskelet Disord. 2008. doi:10.1186/1471-2474-9-42.

83. Miekisiak G, Banach M, Kiwic G, Kubaszewski L, Kaczmarczyk J, et al. Reliability and validity of the Polish version of the Core Outcome Measures Index for the neck. Eur Spine J. 2014. doi:10.1007/s00586-013-3129-2.

84. Monticone M, Vernon H, Brunati R, Rocca B, Ferrante S. The NeckPix@: development of an evaluation tool for assessing kinesiophobia in subjects with chronic neck pain. Eur Spine J. 2014. doi:10.1007/s00586-014-3509-2.

85. Smart A. A multi-dimensional model of clinical utility. Int J Qual Health Care. 2006;18:377-82.

86. World Health Organization. The International Classification of Functioning, Disability and Health (ICF). 2001; Available from: http://www.who.int/ classifications/icf/en/ Accessed 10 Feb 2016.

87. Ferreira ML, Borges BM, Rezende IL, Carvalho LP, Soares LP, et al. Are neck pain scales and questionnaires compatible with the international classification of functioning, disability and health? A systematic review. Disabil Rehabil. 2010. doi:10.3109/09638281003611045.

88. Andelic N, Johansen JB, Bautz-Holter E, Mengshoel AM, Bakke E, Roe C. Linking self-determined functional problems of patients with neck pain to the International Classification of Functioning, Disability, and Health (ICF). Patient Prefer Adherence. 2012. doi:10.2147/PPA.S36165.

89. Johansen JB, Roe C, Bakke E, Mengshoel AM, Andelic N. Reliability and responsiveness of the Norwegian version of the Neck Disability Index. Scand J Pain. 2014;5:28-33.

90. Ailliet L, Rubinstein SM, de Vet HCW, van Tulder MW, Terwee CB. Reliability, responsiveness and interpretability of the neck disability index-Dutch version in primary care. Eur Spine J. 2015. doi:10.1007/s00586-014-3359-y.

91. Pereira M, Cruz EB, Domingues L, Duarte S, Carnide F, Fernandes R. Responsiveness and interpretability of the Portuquese version of the Neck Disability Index in patients with chronic neck pain undergoing physiotherapy. Spine (Phila Pa 1976). 2015. doi:10.1097/BRS.0000000000001034.

\section{Submit your next manuscript to BioMed Central and we will help you at every step:}

- We accept pre-submission inquiries

- Our selector tool helps you to find the most relevant journal

- We provide round the clock customer support

- Convenient online submission

- Thorough peer review

- Inclusion in PubMed and all major indexing services

- Maximum visibility for your research

Submit your manuscript at www.biomedcentral.com/submit 